MODELING, IDENTIFICATION AND CONTROL, 1999, vOL. 20, NO. 1, 27-62

doi:10.4173/mic.1999.12

\title{
Nonlinear Oscillations in Coriolis Based Gyroscopes
}

\author{
DAG KRISTIANSEN and OLAV EGELAND $\dagger$
}

Keywords: Cylinder gyroscopes, nonlinear vibrations, energy transfer

In this paper we model and analyze nonlinear oscillations which are known to exist in some Coriolis based gyroscopes due to large amplitude excitation in the drive loop. A detailed derivation of a dynamic model for a cylinder gyroscope which includes geometric nonlinearities is given, and energy transfer between the system's modes are analyzed using perturbation theory and by proposing a simplified model. The model is also simulated, and the results are shown to give an accurate description of the experimental results. This work is done in order to gain a better understanding of the gyroscope's dynamics, and is intended to be a starting point for designing nonlinear observers and vibration controllers for the gyroscope in order to increase the performance.

\section{Introduction}

Coriolis based gyroscopes, in which vibrating cylindrical shells are used as sensing elements, are gyroscopes which possess a number of advantages over conventional spinning wheel gyroscopes. Troublesome bearings are totally eliminated, they have low power requirements, short start up time and very low inherent noise (Langmaid 1996). In addition, if the vibrating cylinder is designed to give a dynamically balanced oscillator, it is known that performance is not, at least to a first order, sensitive to linear acceleration (Fox 1984; Kanani and Burdess 1990).

Although the cylinder gyroscope has many attractive properties, it also introduces some challenges with respect to modeling and control. One of the most important problems is the existence of unwanted superharmonic responses (Nayfeh and Mook 1979) in the drive and sense loop of the gyroscope when the excitation amplitude becomes large. This cannot be explained by linear vibration theory. However, this type of behaviour is well known in the theory of nonlinear oscillations and dynamics (see, e.g., (Nayfeh and Mook 1979; Evan-Iwanowski 1976)).

The dynamics of a cylinder is also known to be very complex. There appears to be no simple rule for determining the spacing of the linear eigenfrequencies as the circumferential and axial wave numbers are varied (Leissa 1973). Also, the natural frequencies of shells do not fall in ascending series with increasing values of the model index (Blevins 1979). In addition, one wants to minimize the size of the gyroscope. This means that it is interesting to investigate the effect of introducing nonlinear observers and vibration controllers in the design.

Langdon (1982) and Fox (1984) analyzed cylinder gyroscopes by representing it as an infinite cylinder. Burdess (1986) investigated the feasibility of a piezoelectric cylinder gyroscope and derived an electromechanical model by using Lagrange's equation. Loveday (1996), Fox (1988) and Shatalov et al. (1996) analyzed the effect of mass imperfections in cylinder gyroscopes. The temperature dependence of zero offset was investigated in Abe et al. (1996). Other references on the subject are, e.g.,

†Department of Engineering Cybernetics, Norwegian University of Science and Technology, N-7034 Trondheim, Norway.

Reprinted, with permission from Kluwer Academic Publishers, from Nonlinear Dynamics. 
Shuta and Abe (1995), Anders and Pearson (1994), Kagawa et al. (1996), Abe et al. $(1992,1996)$. Common for all these references are that they were only analyzing linear models and therefore did not include important nonlinear effects.

In existing industrial gyroscopes, the problem of superharmonic resonances is "solved" by reducing the excitation amplitude, but since the Coriolis acceleration is proportional to this amplitude, this means that the sensitivity of the gyro decreases. However, a more constructive solution may be to introduce vibration damping controllers to reduce the effect of the superharmonic vibrations and therefore be able to increase the performance of existing gyroscopes. From a control design point of view, it is important that the model used for describing the dynamics of the gyroscope just includes the most important effects (in our case the energy transfer), since an unnecessary complicated model will only lead to an unnecessary complicated control design.

Nonlinear vibrations in cylinders have been investigated by a number of researchers. Traditionally, much of this research has been focused on whether the shell behaves as a hard spring or a soft spring, and whether the type of behaviour depends upon the boundary conditions and/or the shell being open or closed (Leissa 1973; Chu 1961; Chen and Babcock 1975). Modal interactions in spherical shells were investigated by Yasuda and Kushida (1984), while the nonlinear forced responses of infinitely long circular cylindrical shells are reported in Nayfeh et al. (1991), Nayfeh and Raouf (1987).

In this paper we extend the method proposed by Burdess (1986) to include geometric nonlinearities in the model. Based on experimental observations, we derive a nonlinear three-mode model of a cylinder gyroscope. This model is then analyzed using the method of multiple-scales (Nayfeh and Mook 1979). We also show how the model can be simplified, but which still incorporate the energy transfer. This is important when designing observers and controllers. The reduced model is then simulated, and the result corresponds well to the theoretical analysis. To the authors' best knowledge, this is the first time someone has proposed a nonlinear model for cylinder gyroscopes.

The paper is organized as follows: In Section 2 we give a short review of the theory of operation of cylinder gyroscopes. A simple experiment is carried out in Section 3. The basic shell equations are given in Section 4. The equations of motion are derived in Section 5 and analyzed in Section 6. A simplified model is proposed in Section 7 and simulated in Section 8. Concluding remarks to this work are given in Section 9.

\section{A short description of cylinder gyroscopes}

The gyroscope analyzed here is based on the use of a uniform vibrating cylinder which is clamped at one end and mounted on a pedestal so that the other end is free to vibrate (see Fig. 1).

The cylinder is assumed to be thin and perfectly axisymmetric. On the outer surface, there are attached eight identical equispaced electrodes, 1 to 8 , and they are electrically connected in pairs: 1 with 5,2 with 6,3 with 7 , and 4 with 8 . A periodic driving force is applied to electrodes 1 and 5 at the resonance frequency of the cylinder such that it is vibrating at the second circumferential mode. This is shown in Fig. 1. The response is measured by electrodes 3 and 7 , and therefore by using a feedback controller, the response can be held at a preset value.

A second set of measurement electrodes are arranged to be set on the $\pm 45^{\circ}$ nodal lines of the forced circumferential mode, and will ideally produce no output as a result of the oscillator vibration.

When the cylinder is rotated about its central axis, the Coriolis inertia force will 

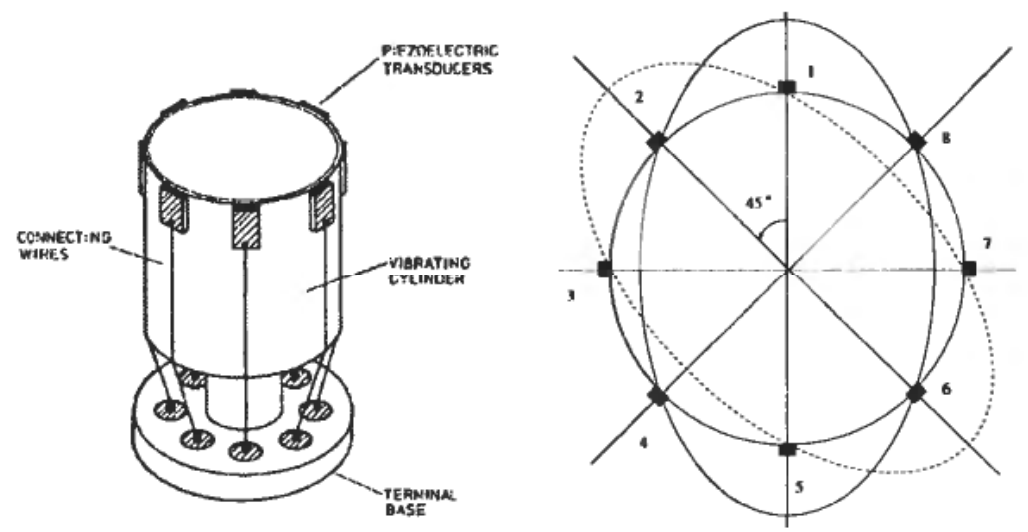

Figure 1. The cylinder gyroscope. The left figure is taken from [15].

generate a secondary motion, as shown by the dotted line in Fig. 1. This new vibration will generate an output on electrodes 2 and 6 which is proportional to the angular rotation velocity. If the voltage applied to electrodes 2 and 6 is provided by introducing negative feedback from electrodes 4 and 8 , it is possible to drive the secondary motion to a null value. This is necessary so that the gyroscope can respond to rapid changes of angular velocity. The voltage applied at electrodes 2 and 6 is taken as a measure of the applied rate of turn.

In order to increase the sensitivity of the gyroscope, one can theoretically do this by increasing the amplitude of the excitation, since the generated Coriolis acceleration is proportional to the excitation amplitude (Fox 1988).

For more information of the theory of operation of the gyroscope and the control strategies, the reader is referred to (Langdon 1982; Fox 1988; Burdess 1986).

\section{Experiments on a non-rotating gyroscope}

In this section we will illustrate by an experiment that when the excitation amplitude becomes large, there will be an energy transfer from the excited mode into higher-frequency modes whose frequencies are determined by the linear natural frequencies of the gyroscope. A signal generator was connected to a cylindrical gyroscope made of steel which attached piezoceramics (Fig. 2). The diameter of the gyroscope was about $15 \mathrm{~mm}$, and the length was about $24 \mathrm{~mm}$. The first three eigenfrequencies of the gyroscope which could be observed by this set-up was at approximately $14.6,43 \cdot 5$, and $61 \cdot 3 \mathrm{kHz}$.

A harmonic excitation with different values of $f_{1}$ and with $\Omega$ adjusted to drive the gyroscope into self-oscillation at the second circumferential mode $\left(\Omega=\bar{\omega}_{1}\right)$ was connected to transducers no. 1 and 5 , and the output from transducer no. 3 and 7 , then no. 2 and 6 was analyzed with an FFT-analyzer. The results are shown in Figs 3-6, and Table 1.

The constant bias and the peak at $14.5 \mathrm{kHz}$ in Figs 5 and 6 is basically due to geometrical errors in the location of the electrodes, and to the fact that the cylinder is not perfectly axisymmetric (Shatalov et al. 1996). This can however be taken care of during the calibration process.

The Fourier spectrum of the excitation signal from the signal generator with $f_{1}=4 \mathrm{~V}$ and $\Omega=\bar{\omega}_{1} \approx 91 \cdot 1 \cdot 10^{3} \mathrm{rad} / \mathrm{s}(14.5 \mathrm{kHz})$ is shown in Fig. 7 .

From this experiment we can conclude that when the amplitude of the excitation 


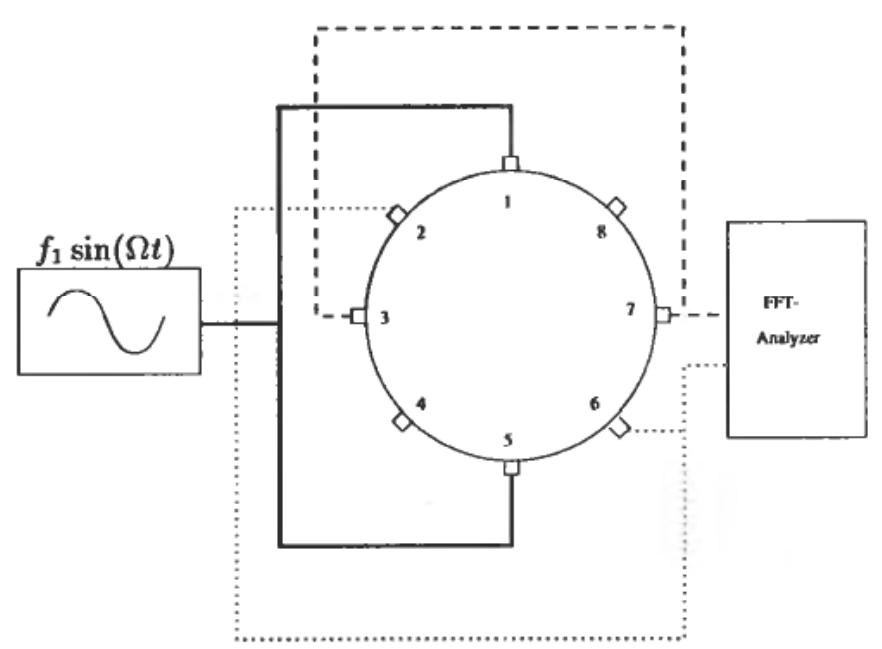

Figure 2. The experimental setup.

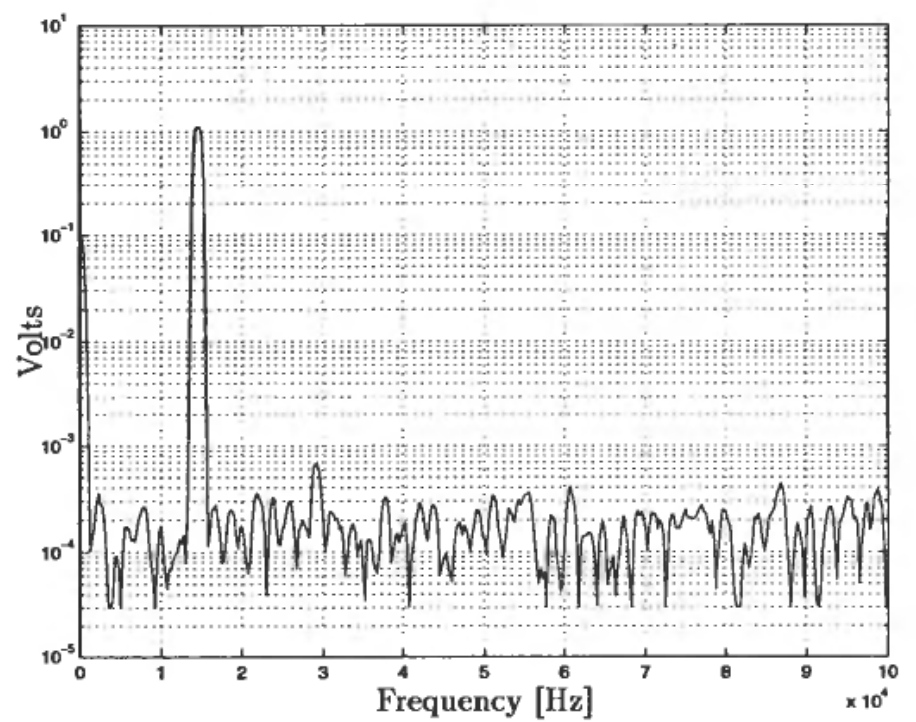

Figure 3. The Fourier spectrum from transducers no. 3 and 7 with $f_{1}=1 \mathrm{~V}$ and $\Omega=\bar{\omega}_{1} \approx 91 \cdot 1 \cdot 10^{3} \mathrm{rad} / \mathrm{s}(14.5 \mathrm{kHz})$.

is large, energy will be transferred up in the frequency spectrum. If these additional peaks are not associated with second circumferential modes, they result in a time-varying bias and are therefore unwanted.

\section{Shell equations}

In this section, we give the basic equations which are used to derive the equations of motion in Section 5.

Consider a thin, cylindrical shell as shown in Fig. 8. The cylinder seen from above with piezoelectric ceramics is shown in Fig. 9. The cylinder is clamped at $x=0$ and 


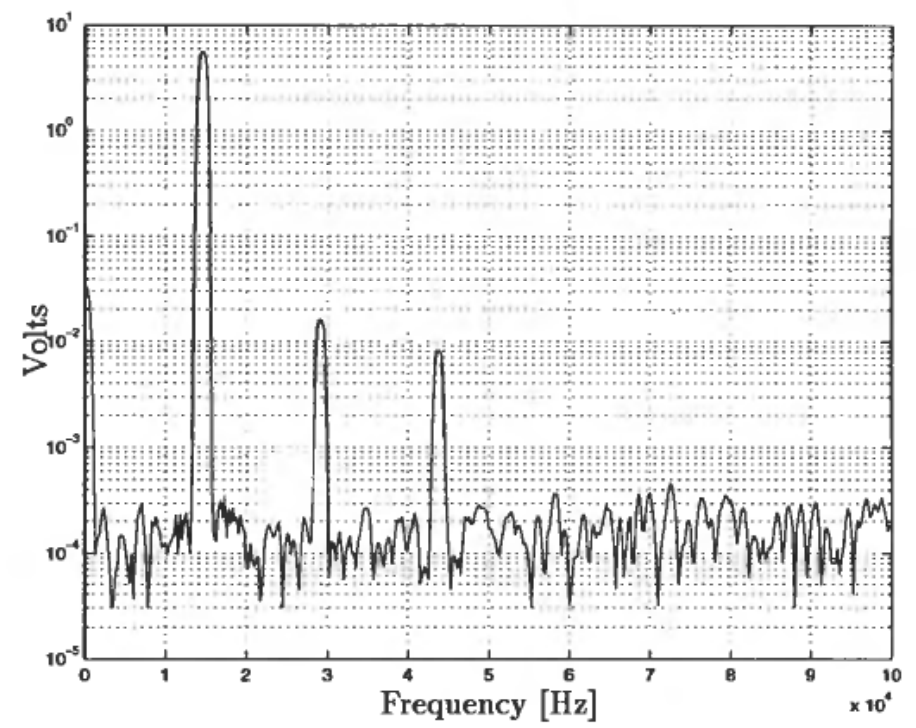

Figure 4. The Fourier spectrum from transducers no. 3 and 7 with $f_{1}=4 \mathrm{~V}$ and $\Omega=\bar{\omega}_{1} \approx 91 \cdot 1 \cdot 10^{3} \mathrm{rad} / \mathrm{s}(14.5 \mathrm{kHz})$.

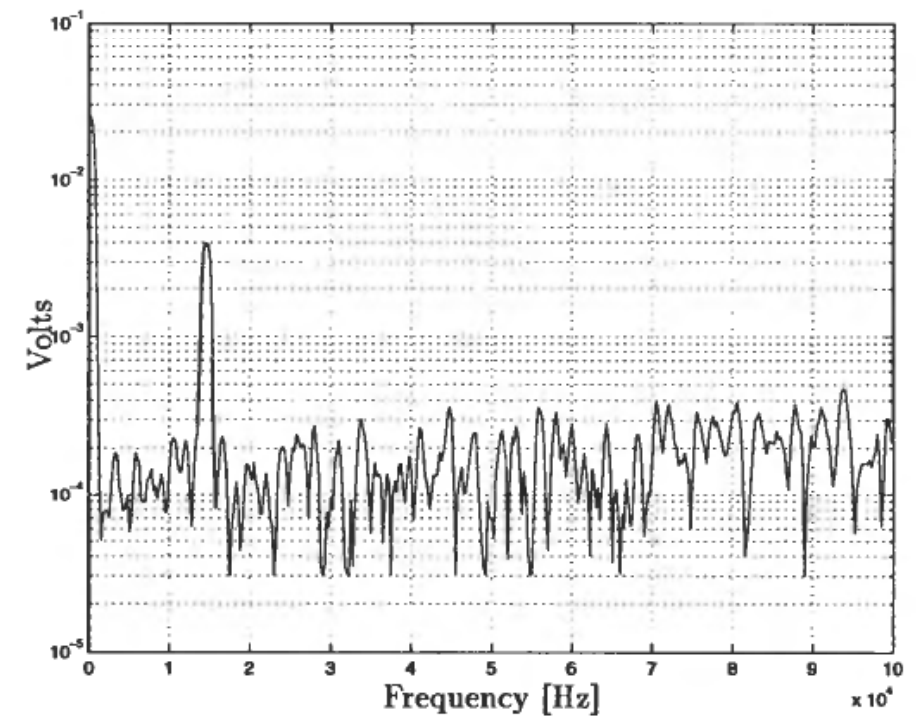

Figure 5. The Fourier spectrum from transducers no. 2 and 6 with $f_{1}=1 \mathrm{~V}$ and $\Omega=\bar{\omega}_{1} \approx 91 \cdot 1 \cdot 10^{3} \mathrm{rad} / \mathrm{s}(14.5 \mathrm{kHz})$.

free at $x=l$. We assume that the radial stress $\sigma_{z z}$, and the shear strains $\varepsilon_{x z}$ and $\varepsilon_{\theta_{z}}$ are negligible, since we are considering a thin cylinder.

There are a number of theories describing the deformations of a shell (Blevins 1979; Leissa 1973). Of the most common theories, Donnell-Mushtari shell theory is considered as the most simple, and Flüegge-Sanders shell theories are generally felt to be most accurate (Blevins 1979). Due to the fact that in the design of control systems, 


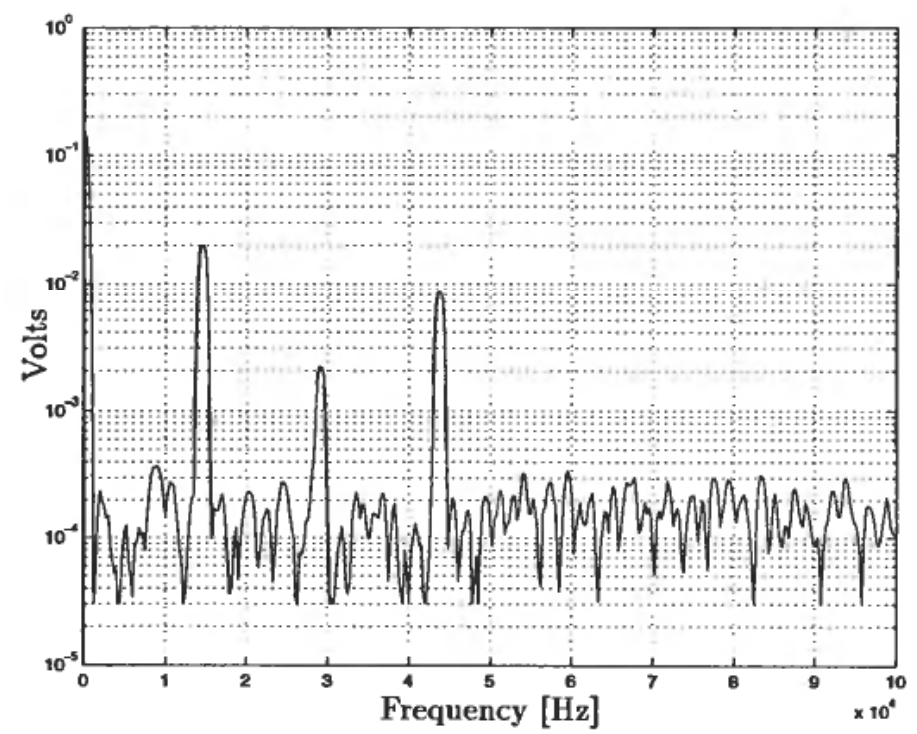

Figure 6. The Fourier spectrum from transducers no. 2 and 6 with $f_{1}=4 \mathrm{~V}$ and $\Omega=\bar{\omega}_{1} \approx 91 \cdot 1 \cdot 10^{3} \mathrm{rad} / \mathrm{s}(14.5 \mathrm{kHz})$.

Table 1. Experimental results with $\Omega=\bar{\omega}_{1}$

\begin{tabular}{llll}
\hline Fig. no. & Electrode no. & $f_{1}$ & Type of response \\
\hline 3 & 3 and 7 & $1 \mathrm{~V}$ & $\begin{array}{l}\text { Except from the constant bias, there is one peak } \\
(\approx 1 \cdot 1 \mathrm{~V}) \text { at } \approx 14.5 \mathrm{kHz}\end{array}$ \\
4 & $4 \mathrm{~V}$ and 7 & $\begin{array}{l}\text { Except from the constant bias, there are three significant } \\
\text { peaks. The first one }(\approx 5.5 \mathrm{~V}) \text { at } \approx 14.5 \mathrm{kHz} \text {, the second } \\
\text { one }(\approx 0.016 \mathrm{~V}) \text { at } \approx 29.25 \mathrm{kHz} \text { and the third one } \\
(\approx 0.0082 \mathrm{mV}) \text { at } \approx 43.75 \mathrm{kHz}\end{array}$ \\
5 & $1 \mathrm{~V}$ & $\begin{array}{l}\text { Except from the constant bias, there is one peak } \\
(\approx 3.9 \mathrm{mV}) \text { at } \approx 14.5 \mathrm{kHz}\end{array}$ \\
6 & $4 \mathrm{~V}$ & $\begin{array}{l}\text { Except from the constant bias, there are three significant } \\
\text { peaks. The first one }(\approx 0.02 \mathrm{~V}) \text { at } \approx 14.5 \mathrm{kHz}, \text { the } \\
\text { second one }(\approx 2.1 \mathrm{mV}) \text { at } \approx 29.25 \mathrm{kHz} \text { and the third } \\
\text { one }(\approx 8.4 \mathrm{mV}) \text { at } \approx 43.75 \mathrm{kHz}\end{array}$ \\
\hline
\end{tabular}

it is common to work with the simplest possible models, we here use the Donnell-Mushtari shell theory. The displacement $\boldsymbol{u}^{2}$ can then be written as

$$
\begin{gathered}
u_{1}^{z}=u_{1}-z \frac{\partial u_{3}}{\partial x} \\
u_{2}^{z}=u_{2}-\frac{z}{R} \frac{\partial u_{3}}{\partial \theta} \\
u_{3}^{z}=u_{3} \\
\mathbb{}
\end{gathered}
$$


where

$$
\left(\begin{array}{l}
u_{1}^{z} \\
u_{2}^{z} \\
u_{3}^{z}
\end{array}\right)=\left(\begin{array}{ccc}
1 & 0 & -z \frac{\partial}{\partial x} \\
0 & 1 & -\frac{z}{R} \frac{\partial}{\partial \theta} \\
0 & 0 & 1
\end{array}\right)\left(\begin{array}{l}
u_{1} \\
u_{2} \\
u_{3}
\end{array}\right)
$$

$$
\boldsymbol{u}^{z}=\left(\begin{array}{l}
u_{1}^{z} \\
u_{2}^{z} \\
u_{3}^{z}
\end{array}\right)
$$

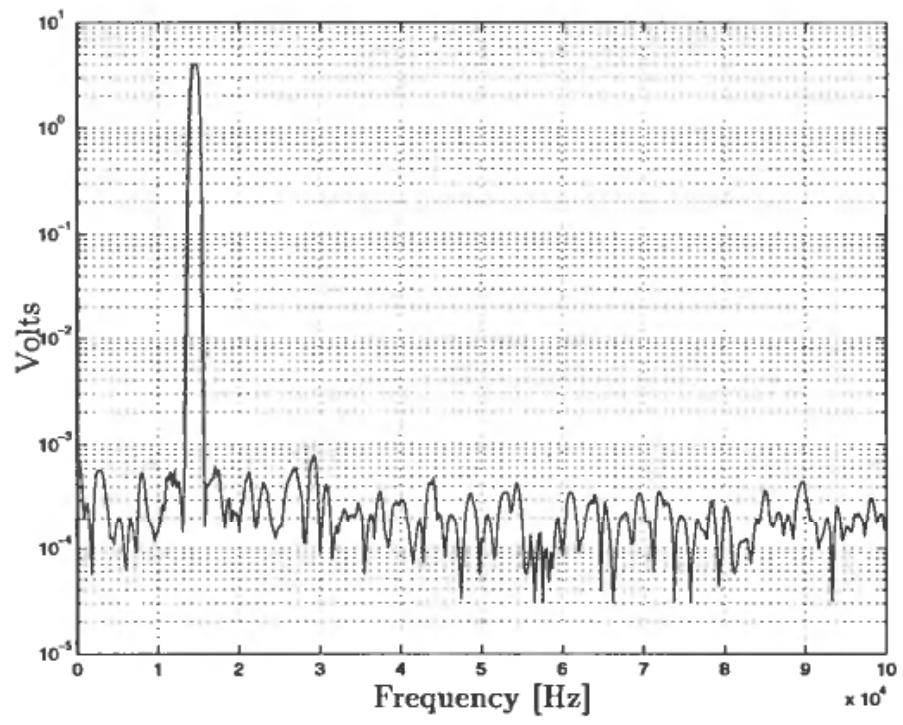

Figure 7. The Fourier spectrum of the excitation signal from the signal generator with $f_{1}=4 \mathrm{~V}$ and $\Omega=\bar{\omega}_{1} \approx 91 \cdot 1 \cdot 10^{3} \mathrm{rad} / \mathrm{s}(14.5 \mathrm{kHz})$.

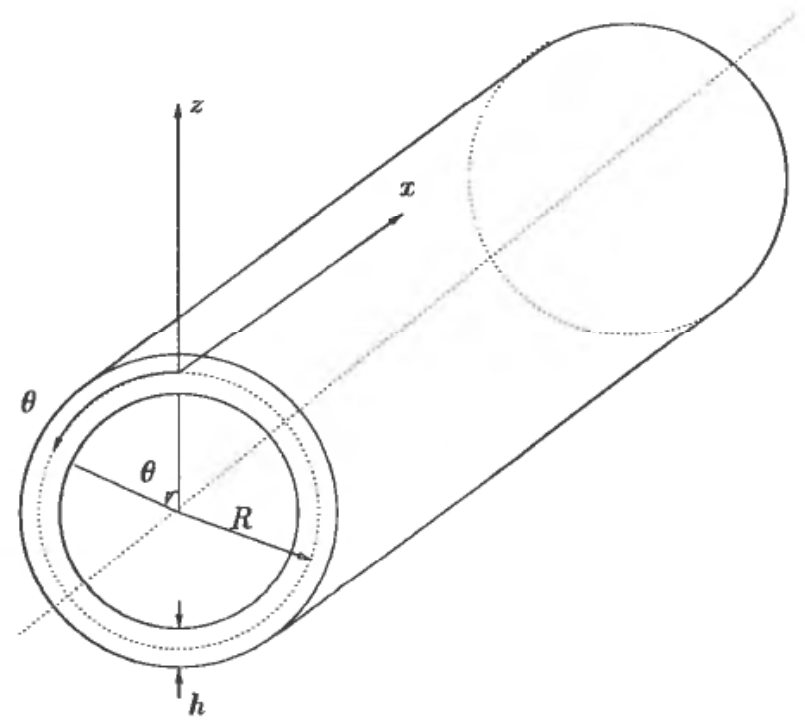

Figure 8. Geometry of a thin cylindrical shell with length $l$. 


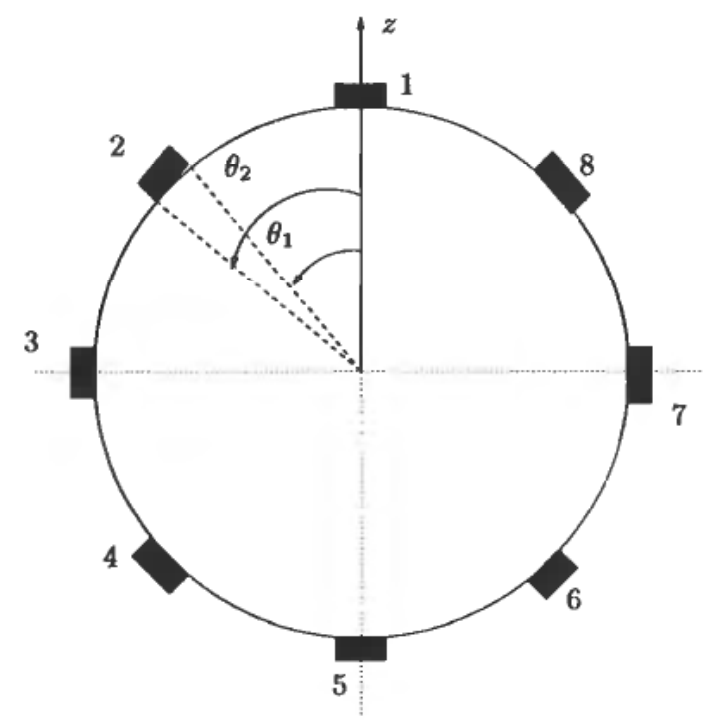

Figure 9. The cylinder with piezoelectric ceramics seen from above.

is the displacement at $z$, and

$$
\boldsymbol{u}=\left(\begin{array}{l}
u_{1} \\
u_{2} \\
u_{3}
\end{array}\right)
$$

is the displacement at the mid-surface respectively in the axial, circumferential and radial direction.

The expressions for the axial $\left(\varepsilon_{x x}\right)$, circumferential $\left(\varepsilon_{00}\right)$, and shear $\left(\varepsilon_{x \theta}\right)$ strains in which the second-order terms are included, can be written as (Leissa 1973; Chu 1961)

$$
\begin{aligned}
& \varepsilon_{x x}=\varepsilon_{11}^{l i n}+z k_{11}+\varepsilon_{11}^{n !} \\
& \varepsilon_{\theta \theta}=\varepsilon_{22}^{l i n}+z k_{22}+\varepsilon_{22}^{n !} \\
& \varepsilon_{x \theta}=\varepsilon_{12}^{l i n}+z k_{12}+\varepsilon_{12}^{n !}
\end{aligned}
$$

where

$$
\begin{aligned}
& \varepsilon_{11}^{l i n}=\frac{\partial u_{1}}{\partial x} \\
& \varepsilon_{22}^{l i n}=\frac{1}{R} \frac{\partial u_{2}}{\partial \theta}+\frac{1}{R} u_{3} \\
& \varepsilon_{12}^{l i n}=\frac{1}{R} \frac{\partial u_{1}}{\partial \theta}+\frac{\partial u_{2}}{\partial x} \\
& \varepsilon_{11}^{n l}=\frac{1}{2}\left(\frac{\partial u_{3}}{\partial x}\right)^{2} \\
& \varepsilon_{22}^{n l}=\frac{1}{2 R^{2}}\left(\frac{\partial u_{3}}{\partial \theta}\right)^{2}
\end{aligned}
$$




$$
\varepsilon_{12}^{n l}=\frac{1}{R} \frac{\partial u_{3}}{\partial x} \frac{\partial u_{3}}{\partial \theta}
$$

and

$$
\left(\begin{array}{l}
k_{11} \\
k_{22} \\
k_{12}
\end{array}\right)=\left(\begin{array}{ccc}
0 & 0 & -\frac{\partial^{2}}{\partial x^{2}} \\
0 & 0 & -\frac{1}{R^{2}} \frac{\partial^{2}}{\partial \theta^{2}} \\
0 & 0 & -\frac{2}{R} \frac{\partial^{2}}{\partial x \partial \theta}
\end{array}\right)\left(\begin{array}{l}
u_{1} \\
u_{2} \\
u_{3}
\end{array}\right)
$$

For an isotropic shell, the expressions for the axial $\left(\sigma_{x x}\right)$, circumferential $\left(\sigma_{\theta \theta}\right)$, and shear $\left(\sigma_{x 0}\right)$ stresses is given by

$$
\left(\begin{array}{l}
\sigma_{x x} \\
\sigma_{\theta 0} \\
\sigma_{x \theta}
\end{array}\right)=\left(\begin{array}{ccc}
c_{11} & c_{12} & 0 \\
c_{12} & c_{11} & 0 \\
0 & 0 & c_{33}
\end{array}\right)\left(\begin{array}{l}
\varepsilon_{x x} \\
\varepsilon_{\theta \theta} \\
\varepsilon_{x \theta}
\end{array}\right)
$$

where $c_{i j}$ is elastic coefficients.

The constitutive properties of the piezoceramics, can be written for electrode $i$ as (Hagood et al. 1990):

$$
\left(\begin{array}{c}
D_{x}^{i} \\
D_{0}^{i} \\
D_{z}^{i} \\
\hline \hat{\sigma}_{x x}^{i} \\
\hat{\sigma}_{\theta 0}^{i} \\
\hat{\sigma}_{x 0}^{i}
\end{array}\right)=\left(\begin{array}{ccc|ccc}
\epsilon_{1}^{s} & 0 & 0 & 0 & 0 & 0 \\
0 & \epsilon_{1}^{s} & 0 & 0 & 0 & 0 \\
0 & 0 & \epsilon_{3}^{s} & e_{31} & e_{31} & 0 \\
\hline 0 & 0 & -e_{31} & c_{11}^{E} & c_{12}^{E} & 0 \\
0 & 0 & -e_{31} & c_{12}^{E} & c_{11}^{E} & 0 \\
0 & 0 & 0 & 0 & 0 & c_{66}^{E}
\end{array}\right)\left(\begin{array}{c}
E_{x}^{i} \\
E_{0}^{i} \\
E_{z}^{i} \\
\hline \hat{\varepsilon}_{x x}^{i} \\
\hat{\varepsilon}_{\theta 0}^{i} \\
\hat{\varepsilon}_{x 0}^{i}
\end{array}\right)
$$

where $D_{n}^{i}$ and $E_{n}^{i}$ are respectively the electric displacement and the electric field in the $n$th direction. $\epsilon_{j}^{s}$ are dielectic constants measured at constant strain, $e_{j k}$ are piezoelectric stiffness constants, $c_{j k}^{E}$ are piezoelectric stiffness constants measured at constant electric field and

$$
\begin{aligned}
& \hat{\boldsymbol{\varepsilon}}_{x x}^{i}=\hat{\boldsymbol{\varepsilon}}_{11}^{\text {in }}+z \hat{k}_{11} \\
& \hat{\boldsymbol{\varepsilon}}_{\theta 0}^{i}=\hat{\boldsymbol{\varepsilon}}_{22}^{\text {lin }}+z \hat{k}_{22} \\
& \hat{\boldsymbol{\varepsilon}}_{x \theta}^{i}=\hat{\boldsymbol{\varepsilon}}_{12}^{\text {lin }}+z \hat{k}_{12}
\end{aligned}
$$

We will study electrodes made of polarized PZT, thus

$$
\begin{aligned}
& D_{x}^{i}=D_{0}^{i}=0 \\
& D_{z}^{i}=\epsilon_{3}^{s} E_{z}^{i}+e_{31}\left(\hat{\varepsilon}_{x x}^{i}+\hat{\varepsilon}_{0 \theta}^{i}\right)
\end{aligned}
$$

Following the same arguments as (Burdess 1986), it can be shown that the electric field under the $i$ th electroded region can be written as

$$
\begin{aligned}
E_{z}^{i} & =\frac{1}{\epsilon_{3}^{s}}\left(D_{z}^{i}-e_{31}\left(\hat{\varepsilon}_{x x}^{i}+\hat{\varepsilon}_{\theta 0}^{i}\right)\right) \\
& =\frac{1}{\epsilon_{3}^{s}}\left(\left[e_{31}\left(\hat{\varepsilon}_{11}^{i}+\hat{\varepsilon}_{22}^{i}\right)-\frac{\epsilon_{3}^{s}}{h_{p}} V_{i}\right]-e_{31}\left(\hat{\varepsilon}_{11}^{i}+z \hat{k}_{11}+\hat{\varepsilon}_{22}^{i}+z \hat{k}_{22}^{i}\right)\right)
\end{aligned}
$$


where $V_{i}$ is the potential difference between the outer electrodes and the inner earthed electrodes (Burdess 1986).

\subsection{Kinetic energy}

We assume that the mass of the piezoelectric transducers is negligible compared to the mass of the cylinder. The kinetic energy is then given as

$$
\begin{aligned}
T= & \frac{1}{2} \rho \int_{0}^{l} \int_{0}^{2 \pi} \int_{-\frac{h}{2}}^{\frac{h}{2}}\left[\left(\dot{u}_{1}^{z}\right)^{2}+\left(\dot{u}_{2}^{z}\right)^{2}+\left(\dot{u}_{3}^{z}\right)^{2}\right] R d z d \theta d x \\
= & \frac{1}{2} \rho \int_{0}^{l} \int_{0}^{2 \pi} \int_{-\frac{h}{2}}^{\frac{h}{2}}\left[\left(\dot{u}_{1}-z \frac{\partial^{2} u_{3}}{\partial x \partial t}\right)^{2}+\left(\dot{u}_{2}-\frac{z}{R} \frac{\partial^{2} u_{3}}{\partial 0 \partial t}\right)^{2}+\dot{u}_{3}^{2}\right] R d z d 0 d x \\
= & \frac{1}{2} \rho \int_{0}^{l} \int_{0}^{2 \pi} \int_{-\frac{h}{2}}^{\frac{h}{2}}\left[\dot{u}_{1}^{2}-2 z \dot{u}_{1} \frac{\partial^{2} u_{3}}{\partial x \partial t}\right)+z^{2}\left(\frac{\partial^{2} u_{3}}{\partial x \partial t}\right)^{2}+\dot{u}_{2}^{2} \\
& \left.+\left(\frac{z}{R}\right)^{2}\left(\frac{\partial^{2} u_{3}}{\partial \theta \partial t}\right)^{2}-2 \frac{z}{R} \frac{\partial^{2} u_{3}}{\partial 0 \partial t} \dot{u}_{2}+\dot{u}_{3}^{2}\right] R d z d 0 d x
\end{aligned}
$$

Integrating over $z$, and neglecting terms associated with rotary inertia and terms of order $\left(\frac{h}{R}\right)^{2}$ and higher in accordance with thin shell theory, the kinetic energy can be written as

$$
T=\frac{1}{2} \rho h R \int_{0}^{l} \int_{0}^{2 \pi}\left[\dot{u}_{1}^{2}+\dot{u}_{2}^{2}+\dot{u}_{3}^{2}\right] d 0 d x
$$

\subsection{Potential energy}

The electric potential energy is given as

$$
W=\frac{1}{2} \sum_{i=1}^{8} \int_{0}^{l p} \int_{\theta_{i}}^{\theta_{i+1}} \int_{-\frac{h p}{2}}^{\frac{h p}{2}} E_{z}^{i} D_{z}^{i}\left(R+\frac{1}{2}\left(h+h_{p}\right)\right) d z d \theta d x
$$

The mechanical strain energy can be written as

$$
\begin{aligned}
U= & \frac{1}{2} \int_{0}^{l} \int_{0}^{2 \pi} \int_{-\frac{h}{2}}^{\frac{h}{2}}\left[\sigma_{x x} \varepsilon_{x x}+\sigma_{00} \varepsilon_{\theta \theta}+\sigma_{x \theta} \varepsilon_{x \theta}\right] R d z d 0 d x \\
& +\frac{1}{2} \sum_{i=1}^{8} \int_{0}^{l p} \int_{0_{i}}^{\theta_{i+1}} \int_{-\frac{h p}{2}}^{\frac{h p}{2}}\left[\hat{\sigma}_{x x}^{i} \hat{\varepsilon}_{x x}^{i}+\hat{\sigma}_{0 \theta}^{i} \hat{\varepsilon}_{\theta \theta}^{i}+\hat{\sigma}_{x \theta}^{i} \hat{\varepsilon}_{x \theta}^{i}\right]\left(R+\frac{1}{2}\left(h+h_{p}\right)\right) d z d 0 d x
\end{aligned}
$$

Following the same arguments as in (Burdess 1986), it can be shown that $W-U$ can be written as

$$
\begin{aligned}
W-U= & \left(\frac{\epsilon_{3}^{s} R}{h_{p}} V_{i}-2 e_{31} R\left(\hat{\varepsilon}_{11}^{i} \hat{\varepsilon}_{22}^{i}\right)\right) V_{i}-\frac{e_{31}^{2} R h_{p}^{3}}{12 \epsilon_{3}^{s}}\left(\hat{k}_{11}^{i}+\hat{k}_{22}^{i}\right)^{2} \\
& -c_{11}^{E} R h_{p}\left(\left(\hat{\varepsilon}_{11}^{i}+\hat{\varepsilon}_{22}^{i}\right)^{2}-2\left(\frac{c_{11}^{E}-c_{12}^{E}}{c_{11}^{E}}\right)\left(\hat{\varepsilon}_{11}^{i} \hat{\varepsilon}_{22}^{i}\right.\right. \\
& \left.\left.-\frac{1}{2}\left(\frac{c_{66}^{E}}{c_{11}^{E}-c_{12}^{E}}\right)\left(\hat{\varepsilon}_{12}^{i}\right)^{2}\right)\right)-\frac{c_{11}^{E} h_{p}^{3} R}{12}\left(\left(\hat{k}_{11}^{i}+\hat{k}_{22}^{i}\right)^{2}\right. \\
& \left.-2\left(\frac{c_{11}^{E}-c_{12}^{E}}{c_{11}^{E}}\right)\left(\hat{k}_{11}^{i}+\hat{k}_{22}^{i}-\frac{1}{2}\left(\frac{c_{66}^{E}}{c_{11}^{E}-c_{12}^{E}}\right)\left(\hat{k}_{12}^{i}\right)^{2}\right)\right)
\end{aligned}
$$




$$
\begin{aligned}
& -\frac{1}{2} \int_{0}^{l} \int_{0}^{2 \pi} \int_{-\frac{h}{2}}^{\frac{h}{2}} c_{11}\left[\left(\varepsilon_{x x}+\varepsilon_{\theta 0}\right)^{2}-2\left(\frac{c_{11}-c_{12}}{c_{11}}\right)\left[\varepsilon_{x x} \varepsilon_{0 \theta}\right.\right. \\
& \left.\left.-\frac{1}{2}\left(\frac{c_{33}}{c_{11}-c_{12}}\right)\left(\varepsilon_{x \theta}\right)^{2}\right]\right] R d z d 0 d x
\end{aligned}
$$

\section{Equations of motion}

The Lagrangian $(L)$ for the gyroscope is given by using (22) and (25):

$$
\begin{aligned}
& L=T+W-U \\
& =\frac{1}{2} \int_{0}^{l} \int_{0}^{2 \pi}\left[\rho R h\left(\dot{u}_{1}^{2}+\dot{u}_{2}^{2}+\dot{u}_{3}^{2}\right)\right. \\
& -c_{11} R h\left(\left(\varepsilon_{11}^{l i n}+\varepsilon_{11}^{n l}+\varepsilon_{22}^{l i n}+\varepsilon_{22}^{n l}\right)^{2}-2\left(\frac{c_{11}-c_{12}}{c_{11}}\right)\left(\left(\varepsilon_{11}^{l i n}+\varepsilon_{11}^{n l}\right)\left(\varepsilon_{22}^{l i n}+\varepsilon_{22}^{n l}\right)\right.\right. \\
& \left.\left.-\frac{1}{2}\left(\frac{c_{33}}{c_{11}-c_{12}}\right)\left(\varepsilon_{12}^{\operatorname{lin}}+\varepsilon_{12}^{n l}\right)^{2}\right)\right) \\
& \left.-\frac{c_{11} h^{3} R}{12}\left(\left(k_{11}+k_{22}\right)^{2}-2\left(\frac{c_{11}-c_{12}}{c_{11}}\right)\left(k_{11} k_{22}-\frac{1}{2}\left(\frac{c_{33}}{c_{11}-c_{12}}\right) k_{12}^{2}\right)\right)\right] d 0 d x \\
& +\frac{1}{2} \sum_{i=1}^{8} \int_{0}^{l p} \int_{\theta_{i}}^{\theta_{i+1}}\left[\left(\frac{\epsilon_{3}^{s} R}{h_{p}} V_{i}-2 e_{31} R\left(\hat{\varepsilon}_{11}^{i}+\hat{\varepsilon}_{22}^{i}\right)\right) V_{i}\right. \\
& -\frac{e_{31}^{2} R h_{p}^{3}}{12 \epsilon_{3}^{s}}\left(\hat{k}_{11}^{i}+\hat{k}_{22}^{i}\right)^{2}-c_{11}^{E} R h_{p}\left(\left(\hat{\varepsilon}_{11}^{i}+\hat{\varepsilon}_{22}^{i}\right)^{2}\right. \\
& \left.-2\left(\frac{c_{11}^{E}-c_{12}^{E}}{c_{11}^{E}}\right)\left(\hat{\varepsilon}_{11}^{i} \hat{\varepsilon}_{22}^{i}-\frac{1}{2}\left(\frac{c_{66}^{E}}{c_{11}^{E}-c_{12}^{E}}\right)\left(\hat{\varepsilon}_{12}^{i}\right)^{2}\right)\right) \\
& \left.-\frac{c_{11}^{E} h_{p}^{3} R}{12}\left(\left(\hat{k}_{11}^{i}+\hat{k}_{22}^{i}\right)^{2}-2\left(\frac{c_{11}^{E}-c_{12}^{E}}{c_{11}^{E}}\right)\left(\hat{k}_{11}^{i} \hat{k}_{22}^{i}-\frac{1}{2}\left(\frac{c_{66}^{E}}{c_{11}^{E}-c_{12}^{E}}\right)\left(\hat{k}_{12}^{i}\right)^{2}\right)\right)\right] d 0 d x
\end{aligned}
$$

Based on the results in Section 3, and due to the configuration of the piezoelectric transducers and the shape of the circumferential modes, we assume that the cylinder motion can be expressed as a 3-mode discretization of the continuous system in the form

$$
u(t, x, \theta)=\sum_{n=1}^{3} \eta_{n}(t)\left(\begin{array}{l}
U_{1 n}(x) \cos 2 n \theta \\
U_{2 n}(x) \sin 2 n \theta \\
U_{3 n}(x) \cos 2 n \theta
\end{array}\right)
$$

where $U_{m n}(x)$ are the linear undamped axial modes of the system without piezoceramics and are derived in Appendix A. $\eta_{n}(t)$ represent the observed modes in Figures 4 and 6 and can be treated as generalized coordinates.

Introduce $\psi=\left|\psi_{k}\right|$, which is given as

$$
\begin{array}{ll}
\psi_{k}=0, & \text { for } k=0, \\
\psi_{k}=0_{k+1}-0_{k}, & \text { for } k=1, \ldots, 8
\end{array}
$$


and let $\eta_{1}, \eta_{2}$ and $\eta_{3}$ be associated with electrode $1,3,5,7$. Then by calculating $L$ by inserting (27) into (26) (see Appendix B) and by using Lagrange's equation in the form

$$
\frac{d}{d t}\left(\frac{\partial L}{\partial \dot{\eta}_{i}}\right)-\frac{\partial L}{\partial \eta_{i}}=0
$$

and adding viscous damping, the differential equations for $\eta_{n}(t)$ can be written as (using $\left.\Omega \approx \bar{\omega}_{1}\right)$

$$
\begin{aligned}
\ddot{\eta}_{1}+2 \bar{\mu}_{1} \dot{\eta}_{1}+\bar{\omega}_{1}^{2} \eta_{1} & =\alpha_{11} \eta_{1}^{3}+\alpha_{12} \eta_{1} \eta_{2}^{2}+\alpha_{13} \eta_{1} \eta_{3}^{2}+\alpha_{14} \eta_{1}^{2} \eta_{3}+\alpha_{15} \eta_{1} \eta_{2}+\alpha_{16} \eta_{2}^{2} \eta_{3} \\
& +\alpha_{17} \eta_{2} \eta_{3}+F_{1} \cos \left(\Omega t+\tau_{1}\right) \\
\ddot{\eta}_{2}+2 \bar{\mu}_{2} \dot{\eta}_{2}+\bar{\omega}_{2}^{2} \eta_{2} & =\alpha_{21} \eta_{2}^{3}+\alpha_{22} \eta_{1}^{2} \eta_{2}+\alpha_{23} \eta_{2} \eta_{3}^{2}+\alpha_{24} \eta_{1}^{2}+\alpha_{25} \eta_{1} \eta_{2} \eta_{3}+\alpha_{26} \eta_{1} \eta_{3} \\
\ddot{\eta}_{3}+2 \bar{\mu}_{3} \dot{\eta}_{3}+\bar{\omega}_{3}^{2} \eta_{3} & =\alpha_{31} \eta_{3}^{3}+\alpha_{32} \eta_{1}^{2} \eta_{3}+\alpha_{33} \eta_{2}^{2} \eta_{3}+\alpha_{34} \eta_{1}^{3}+\alpha_{35} \eta_{1} \eta_{2}^{2}+\alpha_{36} \eta_{1} \eta_{2}
\end{aligned}
$$

where $\bar{\mu}_{i}=\frac{\mu_{i}}{2 \rho R}, \boldsymbol{F}_{i}=\boldsymbol{\beta}_{i} \overline{\boldsymbol{F}}, \omega_{n}$ are the linear natural frequencies associated with $\eta_{n}$ without piezoelectric transducers and

$$
\begin{aligned}
& \bar{\omega}_{1}^{2}=\omega_{1}^{2}+\frac{e_{31}^{2} h_{r}^{3} \psi \int_{0}^{l}\left(\left(\frac{d^{2} U_{31}}{d x^{2}}\right)-\frac{4}{R^{2}} U_{31}\right)^{2} d x}{3 \pi \rho h \epsilon_{3}^{s} \int_{0}^{i}\left(U_{11}^{2}+U_{21}^{2}+U_{31}^{2}\right) d x} \\
& \bar{\omega}_{2}^{2}=\omega_{2}^{2}+\frac{e_{31}^{2} h_{p}^{3} \psi(4 \psi+\sin 4 \psi) \int_{\delta}^{l p}\left(\frac{d^{2} U_{32}}{d x^{2}}-\frac{16}{R^{2}} U_{32}\right)^{2} d x}{12 \epsilon_{3}^{s} \rho h \pi \int_{0}^{l}\left(U_{12}^{2}+U_{22}^{2}+U_{32}^{2}\right) d x} \\
& \bar{\omega}_{3}^{2}=\omega_{3}^{2}+\frac{e_{31}^{2} h_{p}^{3} \psi \int_{0}^{1}\left(\frac{d^{2} U_{33}}{d x^{2}}-\frac{36}{R^{2}} U_{33}\right)^{2} d x}{3 \epsilon_{3}^{3} \rho h \pi \int_{0}^{1}\left(U_{13}^{2}+U_{23}^{2}+U_{33}^{2}\right) d x} \\
& \beta_{1}=\frac{e_{31} R \sin 2 \psi\left(\frac{1}{2} \int_{\delta}^{l}\left(\frac{d U_{11}}{d x}\right) d x+\frac{1}{R} \int_{0}^{l} U_{21} d x+\frac{1}{2 R} \int_{\delta}^{l} U_{31} d x\right)}{\rho R h \pi \int_{0}^{l}\left(U_{11}^{2}+U_{21}^{2}+U_{31}^{2}\right) d x} \\
& \beta_{2}=\frac{e_{31} R \sin 4 \psi\left(\frac{1}{4} \int_{\delta}^{l}\left(\frac{d U_{12}}{d x}\right) d x+\frac{1}{k} \int_{\delta}^{l g} U_{22} d x+\frac{1}{4 R} \int^{l} U_{32} d x\right)}{\rho R h \pi \int_{0}^{l}\left(U_{12}^{2}+U_{22}^{2}+U_{32}^{2}\right) d x} \\
& \beta_{3}=\frac{e_{31} R \sin 6 \psi\left(\frac{1}{6} \int_{\delta}^{l}\left(\frac{d U_{13}}{d x}\right) d x+\frac{1}{R} \int^{l p} U_{23} d x+\frac{1}{6 R} \int_{\delta}^{l p} U_{33} d x\right)}{\rho R h \pi \int_{0}^{1}\left(U_{13}^{2}+U_{23}^{2}+U_{33}^{2}\right) d x} \\
& \bar{F} \cos \left(\Omega t+\tau_{1}\right)=\sum_{i=1}^{4}(-1)^{i+1} V_{2 i-1}
\end{aligned}
$$

and the $\alpha_{i j}$ are defined in Appendix B.

\section{Perturbation analysis}

In this section, we will analyze the internal resonances in the system using the method of multiple scales. A necessary condition for internal resonance is that the linear natural frequencies $\omega_{i}$ are commensurable, i.e., that there exist integer constants $k_{i}$, such that $k_{1} \omega_{1}+\cdots+k_{n} \omega_{n} \approx 0$.

We can neglect the cubic nonlinearities in (30)-(32) since they do not appear in the first-order approximation (Nayfeh and Mook 1979). (30)-(32) can then be written as 


$$
\begin{aligned}
& \ddot{\eta}_{1}+2 \bar{\mu} \dot{\eta}_{1}+\bar{\omega}_{1}^{2} \eta_{1}=\alpha_{15} \eta_{1} \eta_{2}+\alpha_{17} \eta_{2} \eta_{3}+F_{1} \cos \left(\Omega t+\tau_{1}\right) \\
& \ddot{\eta}_{2}+2 \bar{\mu} \dot{\eta}_{2}+\bar{\omega}_{2}^{2} \eta_{2}=\alpha_{24} \eta_{1}^{2}+\alpha_{26} \eta_{1} \eta_{3} \\
& \ddot{\eta}_{3}+2 \bar{\mu} \dot{\eta}_{3}+\bar{\omega}_{3}^{2} \eta_{3}=\alpha_{36} \eta_{1} \eta_{2}
\end{aligned}
$$

To express the nearness of $\Omega$ to $\bar{\omega}_{1}$, we introduce the detuning parameter $\xi_{1}$ where

$$
\Omega=\bar{\omega}_{1}+\epsilon \xi_{1}
$$

and $\epsilon$ is a small dimensionless parameter and a measure of the response amplitudes.

We seek a first-order uniform expansion by using the method of multiple-scales (Nayfeh and Mook 1979) in the form

$$
\begin{aligned}
& \eta_{1}=\epsilon \eta_{11}\left(T_{0}, T_{1}\right)+\epsilon^{2} \eta_{12}\left(T_{0}, T_{1}\right)+\ldots \\
& \eta_{2}=\epsilon \eta_{21}\left(T_{0}, T_{1}\right)+\epsilon^{2} \eta_{22}\left(T_{0}, T_{1}\right)+\ldots \\
& \eta_{3}=\epsilon \eta_{31}\left(T_{0}, T_{1}\right)+\epsilon^{2} \eta_{32}\left(T_{0}, T_{1}\right)+\ldots
\end{aligned}
$$

where

$$
T_{n}=\epsilon^{n} t, \text { for } n=0,1,2, \cdots
$$

It follows that the derivatives with respect to $t$ become expansions in terms of the partial derivatives with respect to $T_{n}$ according to

$$
\begin{gathered}
\frac{d}{d t}=\frac{d T_{0}}{d t} \frac{\partial}{\partial T_{0}}+\frac{d T_{1}}{d t} \frac{\partial}{\partial T_{1}}+\cdots=D_{0}+\epsilon D_{1}+\cdots \\
\frac{d^{2}}{d t^{2}}=D_{0}^{2}+2 \epsilon D_{0} D_{1}+\epsilon^{2}\left(D_{1}^{2}+2 D_{0} D_{2}\right)+\cdots
\end{gathered}
$$

where $D_{n}=\frac{\partial}{\partial T_{n}}$.

In order to have the damping, the nonlinear terms and the forcing term appear in the same perturbation equations, we scale the damping coefficient by letting $\bar{\mu}=\epsilon \hat{\mu}$ and $F_{1}=\epsilon^{2} f_{1}$. Substituting (44)-(46) into (40)-(42) and equating coefficients of like powers of $\epsilon$, we get

\section{Order $\epsilon$}

$$
\begin{aligned}
& D_{0}^{2} \eta_{11}+\bar{\omega}_{1}^{2} \eta_{11}=0 \\
& D_{0}^{2} \eta_{21}+\bar{\omega}_{2}^{2} \eta_{21}=0 \\
& D_{0}^{2} \eta_{31}+\bar{\omega}_{3}^{2} \eta_{31}=0
\end{aligned}
$$

Order $\epsilon^{2}$

$D_{0}^{2} \eta_{12}+\bar{\omega}_{1}^{2} \eta_{12}=-2 D_{0} D_{1} \eta_{11}-2 \hat{\mu} D_{0} \eta_{11}+\alpha_{15} \eta_{11} \eta_{21}+\alpha_{17} \eta_{21} \eta_{31}+f_{1} \cos \left(\Omega T_{0}+\tau_{1}\right)$

$D_{0}^{2} \eta_{22}+\bar{\omega}_{2}^{2} \eta_{22}=-2 D_{0} D_{1} \eta_{21}-2 \hat{\mu} D_{0} \eta_{21}+\alpha_{24} \eta_{11}^{2}+\alpha_{26} \eta_{11} \eta_{31}$

$D_{0}^{2} \eta_{32}+\bar{\omega}_{3}^{2} \eta_{32}=-2 D_{0} D_{1} \eta_{31}-2 \hat{\mu} D_{0} \eta_{31}+\alpha_{36} \eta_{11} \eta_{21}$

The solutions of (50) can be written as

$$
\eta_{11}=A_{1}\left(T_{1}\right) e^{i \bar{\omega}_{1} T_{0}}+c c
$$




$$
\begin{aligned}
& \eta_{21}=A_{2}\left(T_{1}\right) e^{i \bar{\omega}_{2} T_{0}}+c c \\
& \eta_{31}=A_{3}\left(T_{1}\right) e^{i \bar{\omega}_{3} T_{0}}+c c
\end{aligned}
$$

where $c c$ denotes the complex conjugate of the preceding terms, and $A_{n}$ are unknown complex functions.

Substituting these equations into (51) gives

$$
\begin{aligned}
D_{0}^{2} \eta_{12}+\bar{\omega}_{1}^{2} \eta_{12}= & -2 i \bar{\omega}_{1}\left(D_{1} A_{1} e^{i \bar{\omega}_{1} T_{0}}+\hat{\mu} A_{1} e^{i \bar{\omega}_{1} T_{0}}\right) \\
& +\alpha_{15}\left(A_{1} A_{2} e^{i\left(\bar{\omega}_{1}+\bar{\omega}_{2}\right) T_{0}}+A_{2} \bar{A}_{1} e^{i\left(\bar{\omega}_{2}-\bar{\omega}_{1}\right) T_{0}}\right) \\
& +\alpha_{17}\left(A_{2} A_{3} e^{i\left(\bar{\omega}_{2}+\bar{\omega}_{3}\right) T_{0}}+A_{3} \bar{A}_{2} e^{i\left(\bar{\omega}_{3}-\bar{\omega}_{2}\right) T_{0}}\right) \\
& +\frac{1}{2} f_{1} e^{i\left(\bar{\omega}_{1} T_{0}+\bar{\xi}_{1} T_{1}+\tau_{1}\right)}+c c \\
D_{0}^{2} \eta_{22}+\bar{\omega}_{2}^{2} \eta_{22}= & -2 i \bar{\omega}_{2}\left(D_{1} A_{2} e^{i \bar{\omega}_{2} T_{0}}+\hat{\mu} A_{2} e^{i \bar{\omega}_{2} T_{0}}\right) \\
& +\alpha_{24}\left(A_{1}^{2} e^{\left.2 i \bar{\omega}_{1} T_{0}+A_{1} \bar{A}_{1}\right)}\right. \\
& +\alpha_{26}\left(A_{1} A_{3} e^{i\left(\bar{\omega}_{1}+\bar{\omega}_{3}\right) T_{0}}+A_{3} \bar{A}_{1} e^{i\left(\bar{\omega}_{3}-\bar{\omega}_{1}\right) T_{0}}\right)+c c \\
D_{0}^{2} \eta_{32}+\bar{\omega}_{3}^{2} \eta_{32}= & -2 i \bar{\omega}_{3}\left(D_{1} A_{3} e^{i \bar{\omega}_{3} T_{0}}+\hat{\mu} A_{3} e^{i \overline{\omega_{3}} T_{0}}\right) \\
& +\alpha_{36}\left(A_{1} A_{2} e^{i\left(\bar{\omega}_{1}+\bar{\omega}_{2}\right) T_{0}}+A_{2} \bar{A}_{1} e^{i\left(\bar{\omega}_{2}-\bar{\omega}_{1}\right) T_{0}}\right)+c c
\end{aligned}
$$

6.1. The Case of $2 \bar{\omega}_{1} \neq \bar{\omega}_{2}$ and $3 \bar{\omega}_{1} \neq \bar{\omega}_{3}$

In this case, secular terms will be eliminated if

$$
\begin{aligned}
2 i \bar{\omega}_{1}\left(D_{1} A_{1}+\hat{\mu} A_{1}\right) & =\frac{1}{2 f_{1}} e^{i\left(\xi_{1} T_{1}+\tau_{1}\right)} \\
D_{1} A_{2}+\hat{\mu} A_{2} & =0 \\
D_{1} A_{3}+\hat{\mu} A_{3} & =0
\end{aligned}
$$

whose solutions are

$$
\begin{aligned}
& A_{1}=\frac{1}{2} a_{1} e^{-\hat{\mu} T_{1}+i \varphi_{1}}-\frac{i f_{1}}{4 \bar{\omega}_{1}\left(\hat{\mu}+i \xi_{1}\right)} e^{i\left(\xi_{1} T_{1}+\tau_{1}\right)} \\
& A_{2}=\frac{1}{2} a_{2} e^{-\hat{\mu} T_{1}+i \varphi_{2}} \\
& A_{3}=\frac{1}{2} a_{3} e^{-\hat{\mu} T_{1}+i \varphi_{3}}
\end{aligned}
$$

where $a_{n}$ and $\varphi_{n}$ are constants.

As $t \rightarrow \infty, T_{1} \rightarrow \infty$ :

$$
\begin{aligned}
& A_{1} \rightarrow-\frac{i f_{1}}{4 \bar{\omega}\left(\hat{\mu}+i \xi_{1}\right)} e^{i\left(\xi_{1} T_{1}+\tau_{1}\right)} \\
& A_{2} \rightarrow 0
\end{aligned}
$$




$$
A_{3} \rightarrow 0
$$

Substituting (62) into (44)-(46) and expressing the result in terms of the original variables, we obtain the following steady state response

$$
\begin{aligned}
& \eta_{1}=\frac{F_{1}}{2 \epsilon \bar{\omega} \sqrt{\hat{\mu}^{2}+\xi_{1}^{2}}} \sin \left(\Omega t+\tau_{1}-\gamma_{0}\right)+O\left(\epsilon^{2}\right) \\
& \eta_{2}=0+O\left(\epsilon^{2}\right) \\
& \eta_{3}=0+O\left(\epsilon^{2}\right)
\end{aligned}
$$

where $\gamma_{0}=\arctan \left(\frac{\xi_{1}}{\hat{\mu}}\right)$.

We notice that when there are no internal resonances, the first approximation is not influenced by the nonlinear terms. It is essentially the solution of the linear problem.

\subsection{The Case of $2 \bar{\omega}_{1} \approx \bar{\omega}_{2}$ and $\bar{\omega}_{3} \neq \bar{\omega}_{1}+\omega_{2}$}

To express the nearness of $2 \bar{\omega}_{1}$ to $\bar{\omega}_{2}$, we introduce the detuning parameter $\xi_{2}$ according to

$$
\begin{aligned}
& \bar{\omega}_{2}=2 \bar{\omega}_{1}+\epsilon \xi_{2} \\
& \Downarrow \\
&\left(\bar{\omega}_{2}-\bar{\omega}_{1}\right) T_{0}=\left(2 \bar{\omega}_{1}-\bar{\omega}_{1}+\epsilon \xi_{2}\right) T_{0}=\bar{\omega}_{1} T_{0}+\xi_{2} T_{1} \\
& 2 \bar{\omega}_{\mathrm{i}} T_{0}=\left(\bar{\omega}_{2}-\epsilon \xi_{2}\right) T_{0}=\bar{\omega}_{2} T_{0}-\xi_{2} T_{1}
\end{aligned}
$$

Elimination of secular terms:

$$
\begin{aligned}
-2 i \bar{\omega}_{1}\left(D_{1} A_{1}+\hat{\mu} A_{1}\right)+\alpha_{15} A_{2} \bar{A}_{1} e^{i \xi_{2} T_{1}}+\frac{1}{2} e^{i\left(\xi_{1} T_{1}+\tau_{1}\right)} & =0 \\
-2 i \bar{\omega}_{2}\left(D_{1} A_{2}+\hat{\mu} A_{2}\right)+\alpha_{24} A_{1}^{2} e^{-i \xi_{2} T_{1}} & =0 \\
D_{1} A_{3}+\hat{\mu} A_{3} & =0
\end{aligned}
$$

Let

$$
\begin{aligned}
& A_{1}=\frac{1}{2} a_{1} e^{i \varphi_{1}} \\
& A_{2}=\frac{1}{2} a_{2} e^{i \varphi_{2}}
\end{aligned}
$$

where $a_{n}$ and $\varphi_{n}$ are real functions of $T_{1}$. Substituting these equations into (68) leads to

$$
\begin{array}{r}
a_{1}^{\prime}=-\hat{\mu} a_{1}+\frac{\alpha_{15}}{4 \bar{\omega}_{1}} a_{1} a_{2} \sin \left(\xi_{2} T_{1}+\varphi_{2}-2 \varphi_{1}\right)+\frac{1}{2 \bar{\omega}_{1}} f_{1} \cos \left(\xi_{1} T_{1}+\tau_{1}-\varphi_{1}\right) \\
a_{1} \varphi_{1}^{\prime}=-\frac{\alpha_{15}}{4 \bar{\omega}_{1}} a_{1} a_{2} \cos \left(\xi_{2} T_{1}+\tau_{1}-\varphi_{1}\right)-\frac{1}{2 \bar{\omega}_{1}} \frac{f_{1}}{a_{1}} \cos \left(\xi_{1} T_{1}+\tau_{1}-\varphi_{1}\right)
\end{array}
$$

where the prime denotes the derivative with respect to $T_{1}$. 
Substituting (71) and (72) into (69) leads to

$$
\begin{aligned}
a_{2}^{\prime} & =-\hat{\mu} a_{2}-\frac{\alpha_{24}}{4 \bar{\omega}} a_{1}^{2} \sin \left(\xi_{2} T_{1}+\varphi_{2}-2 \varphi_{1}\right) \\
a_{2} \varphi_{2}^{\prime} & =-\frac{\alpha_{24}}{4 \bar{\omega}_{2}} a_{1}^{2} \cos \left(\xi_{2} T_{1}+\varphi_{2}-2 \varphi_{1}\right)
\end{aligned}
$$

Introduce

$$
\begin{aligned}
& \gamma_{1}=\xi_{2} T_{1}+\varphi_{2}-2 \varphi_{1} \\
& \gamma_{2}=\xi_{1} T_{1}+\tau_{1}-\varphi_{1}
\end{aligned}
$$

Then

$$
\begin{aligned}
\gamma_{1}^{\prime} & =\xi_{2}+\varphi_{2}^{\prime}-2 \varphi_{1}^{\prime} \\
& =\xi_{2}+\left(\frac{\alpha_{15}}{2 \bar{\omega}_{1}} a_{2}-\frac{\alpha_{24}}{4 \bar{\omega}_{2}} \frac{a_{1}^{2}}{a_{2}}\right) \cos \gamma_{1}+\frac{1}{\bar{\omega}_{1}} \frac{a_{1}}{f_{1}} \cos \gamma_{2} \\
\gamma_{2}^{\prime} & =\xi_{1} \div \varphi_{1}^{\prime} \\
& =\xi_{1}+\frac{\alpha_{15}}{4 \bar{\omega}_{1}} a_{2} \cos \gamma_{1}+\frac{1}{2 \bar{\omega}_{1}} \frac{f_{1}}{a_{1}} \cos \gamma_{2}
\end{aligned}
$$

From (77), (78), (43), and (66), we have

$$
\begin{aligned}
\varphi_{1} & =\xi_{1} T_{1}+\tau_{1}-\gamma_{2} \\
& =\left(\Omega-\bar{\omega}_{1}\right) T_{0}+\tau_{1}-\gamma_{2} \\
\varphi_{2} & =\div \xi_{2} T_{1}+\gamma_{1}+2 \varphi \\
& =\left(2 \Omega-\bar{\omega}_{2}\right) T_{0}+2 \tau_{1}+\gamma_{1}-2 \gamma_{2}
\end{aligned}
$$

Then the response in the original variables is:

$$
\begin{aligned}
\eta_{1} & =\epsilon \eta_{11}\left(T_{0}, T_{1}\right)+\epsilon^{2} \eta_{12}\left(T_{0}, T_{1}\right)+\cdots \\
& =\epsilon a_{1} \cos \left(\Omega t+\tau_{1}-\gamma_{2}\right)+O\left(\epsilon^{2}\right) \\
\eta_{2} & =\epsilon \eta_{21}\left(T_{0}, T_{1}\right)+\epsilon^{2} \eta_{22}\left(T_{0}, T_{1}\right)+\cdots \\
& =\epsilon a_{2} \cos \left(2 \Omega t+2 \tau_{1}+\gamma_{1} \div 2 \gamma_{2}\right)+O\left(\epsilon^{2}\right) \\
\eta_{3} & =0+O\left(\epsilon^{2}\right)
\end{aligned}
$$

6.3. The Case of $2 \bar{\omega}_{1} \neq \bar{\omega}_{2}$ and $3 \bar{\omega}_{1} \approx \bar{\omega}_{3}$

To express the nearness of $\bar{\omega}_{3}$ to $\bar{\omega}_{1}+\bar{\omega}_{2}$, we introduce the detuning parameter $\xi_{3}$ according to

$$
\begin{gathered}
\bar{\omega}_{3}-\bar{\omega}_{2}=\bar{\omega}_{1}+\epsilon \xi_{3} \\
\Downarrow \\
\left(\bar{\omega}_{3}-\bar{\omega}_{2}\right) T_{0}=\left(\bar{\omega}_{1}+\epsilon \xi_{3}\right) T_{0}=\bar{\omega}_{1} T_{0}+\xi_{3} T_{1} \\
\left(\bar{\omega}_{3}-\bar{\omega}_{1}\right) T_{0}=\left(\bar{\omega}_{2}+\epsilon \xi_{3}\right) T_{0}=\bar{\omega}_{2} T_{0}+\xi_{3} T_{1}
\end{gathered}
$$

Elimination of secular terms:

$$
2 i \bar{\omega}_{1}\left(D_{1} A_{1}+\hat{\mu} A_{1}\right)=\frac{1}{2} f_{1} e^{i\left(\xi_{1} T_{1}+\tau_{1}\right)}
$$




$$
\begin{aligned}
& D_{1} A_{2}+\hat{\mu} A_{2}=0 \\
& D_{1} A_{3}+\hat{\mu} A_{3}=0
\end{aligned}
$$

which are the same equations as (58). This is in agreement with the general theory that if the system has quadratic nonlinearities, then to first-order an internal resonance can exist if $\bar{\omega}_{m} \approx 2 \bar{\omega}_{k}$ or $\bar{\omega}_{q} \approx \bar{\omega}_{p} \pm \bar{\omega}_{m}$. For a system with cubic nonlinearities, to first order an internal resonance can exist if $\bar{\omega}_{m} \approx 3 \bar{\omega}_{k}$ or $\bar{\omega}_{q} \approx 2 \bar{\omega}_{p} \pm \bar{\omega}_{m}$ or $\bar{\omega}_{q} \approx \bar{\omega}_{p} \pm \bar{\omega}_{m} \pm \bar{\omega}_{k}$ (Nayfeh and Mook 1979).

\subsection{The Case of $2 \bar{\omega}_{1} \approx \bar{\omega}_{2}$ and $3 \bar{\omega}_{1} \approx \bar{\omega}_{3}$}

Elimination of secular terms leads to

$$
\begin{aligned}
-2 i \bar{\omega}_{1}\left(D_{1} A_{1}+\hat{\mu} A_{1}\right)+\alpha_{15} A_{2} \bar{A}_{1} e^{i \xi_{2} T_{1}}+\alpha_{17} A_{3} \bar{A}_{2} e^{i \xi_{3} T_{1}}+\frac{1}{2} f_{1} e^{i\left(\xi_{1} T_{1}+\tau_{1}\right)}=0 \\
-2 i \bar{\omega}_{2}\left(D_{1} A_{2}+\hat{\mu} A_{2}\right)+\alpha_{24} A_{1}^{2} e^{-i \xi_{2} T_{1}}+\alpha_{26} A_{3} \bar{A}_{1} e^{i \xi_{3} T_{1}}=0 \\
-2 i \bar{\omega}_{3}\left(D_{1} A_{3}+\hat{\mu} A_{3}\right)+\alpha_{36} A_{1} A_{2} e^{-i \xi_{3} T_{1}}=0
\end{aligned}
$$

Let

$$
\begin{aligned}
& A_{1}=\frac{1}{2} a_{1} e^{i \varphi_{1}} \\
& A_{2}=\frac{1}{2} a_{2} e^{i \varphi_{2}} \\
& A_{3}=\frac{1}{2} a_{3} e^{i \varphi_{3}}
\end{aligned}
$$

Substituting this into (89), (90), and (91) gives

$$
\begin{aligned}
a_{1}^{\prime}= & -\hat{\mu} a_{1}+\frac{\alpha_{15}}{4 \bar{\omega}_{1}} a_{1} a_{2} \sin \left(\xi_{2} T_{1}+\varphi_{2}-2 \varphi_{1}\right) \\
& +\frac{\alpha_{17}}{4 \bar{\omega}_{1}} a_{2} a_{3} \sin \left(\xi_{3} T_{1}+\varphi_{3}-\varphi_{2}-\varphi_{1}\right)+\frac{1}{2 \bar{\omega}_{1}} f_{1} \sin \left(\xi_{1} T_{1}+\tau_{1}-\varphi_{1}\right) \\
a_{2}^{\prime}= & -\hat{\mu} a_{2}-\frac{\alpha_{24}}{4 \bar{\omega}_{2}} a_{1}^{2} \sin \left(\xi_{2} T_{1}+\varphi_{2}-2 \varphi_{1}\right) \\
& +\frac{\alpha_{26}}{4 \bar{\omega}_{2}} a_{1} a_{3} \sin \left(\xi_{3} T_{1}+\varphi_{3}-\varphi_{1}-\varphi_{2}\right) \\
a_{3}^{\prime}= & -\hat{\mu} a_{3}-\frac{\alpha_{36}}{4 \bar{\omega}_{3}} a_{1} a_{2} \sin \left(\xi_{3} T_{1}+\varphi_{3}-\varphi_{1}-\varphi_{2}\right) \\
a_{1}^{\prime} \varphi_{1}^{\prime}= & -\frac{\alpha_{15}}{4 \bar{\omega}_{1}} a_{1} a_{2} \cos \left(\xi_{2} T_{1}+\varphi_{2}-2 \varphi_{1}\right)-\frac{\alpha_{17}}{4 \bar{\omega}_{1}} a_{2} a_{3} \cos \left(\xi_{3} T_{1}+\varphi_{3}-\varphi_{2}-\varphi_{1}\right) \\
& -\frac{f_{1}}{2 \bar{\omega}_{1}} \cos \left(\xi_{1} T_{1}+\tau_{1}-\varphi_{1}\right)
\end{aligned}
$$


$a_{2} \varphi_{2}^{\prime}=-\frac{\alpha_{24}}{4 \bar{\omega}_{2}} a_{1}^{2} \cos \left(\xi_{2} T_{1}+\varphi_{2}-2 \varphi_{1}\right)-\frac{\alpha_{26}}{4 \bar{\omega}_{2}} a_{1} a_{3} \cos \left(\xi_{3} T_{1}+\varphi_{3}-\varphi_{2}-\varphi_{1}\right)$

$a_{3} \varphi_{3}^{\prime}=-\frac{\alpha_{36}}{4 \bar{\omega}_{3}} a_{1} a_{2} \cos \left(\xi_{3} T_{1}+\varphi_{3}-\varphi_{1}-\varphi_{2}\right)$

Introduce

$$
\begin{aligned}
& \gamma_{1}=\xi_{2} T_{1}+\varphi_{2}-2 \varphi_{1} \\
& \gamma_{2}=\xi_{3} T_{1}+\varphi_{3}-\varphi_{2}-\varphi_{1} \\
& \gamma_{3}=\xi_{1} T_{1}+\tau_{1}-\varphi_{1}
\end{aligned}
$$

Then

$$
\begin{aligned}
\gamma_{1}^{\prime}= & \xi_{2}+\varphi_{2}^{\prime}-2 \varphi_{1}^{\prime} \\
= & \xi_{2}+\left(\frac{\alpha_{15}}{2 \bar{\omega}_{1}} a_{2}-\frac{\alpha_{24}}{4 \bar{\omega}_{2}} \frac{a_{1}^{2}}{a_{2}}\right) \cos \gamma_{1}+\left(\frac{\alpha_{17}}{2 \bar{\omega}_{1}} \frac{a_{2} a_{3}}{a_{1}}-\frac{\alpha_{26}}{4 \bar{\omega}_{2}} \frac{a_{1} a_{3}}{a_{2}}\right) \cos \gamma_{2} \\
& +\frac{1}{\bar{\omega}_{1}} \frac{f_{1}}{a_{1}} \cos \gamma_{3} \\
\gamma_{2}^{\prime}= & \xi_{3}+\varphi_{3}^{\prime}-\varphi_{2}^{\prime}-\varphi_{1}^{\prime} \\
= & \xi_{3}+\left(\frac{\alpha_{24}}{4 \bar{\omega}_{2}} \frac{a_{1}^{2}}{a_{2}}+\frac{\alpha_{15}}{4 \bar{\omega}_{1}} a_{2}\right) \cos \gamma_{1}+\frac{\alpha_{17}}{4 \bar{\omega}_{1}} \frac{a_{2} a_{3}}{a_{1}}+\left(\frac{\alpha_{26}}{4 \bar{\omega}_{2}} \frac{a_{1} a_{3}}{a_{2}}-\frac{\alpha_{36}}{4 \bar{\omega}_{3}} \frac{a_{1} a_{2}}{a_{3}}\right) \cos \gamma_{2} \\
& +\frac{1}{2 \bar{\omega}_{1}} \frac{f_{1}}{a_{1}} \cos \gamma_{3} \\
\gamma_{3}^{\prime}= & \xi_{1}-\varphi_{1}^{\prime} \\
= & \xi_{1}+\frac{\alpha_{15}}{4 \bar{\omega}_{1}} a_{2} \cos \gamma_{1}+\frac{\alpha_{17}}{4 \bar{\omega}_{1}} \frac{a_{2} a_{3}}{a_{1}} \cos \gamma_{2}+\frac{1}{2 \bar{\omega}_{1}} \frac{f_{1}}{a_{1}} \cos \gamma_{3}
\end{aligned}
$$

Since

$$
\begin{aligned}
\varphi_{1} & =\xi_{1} T_{1}+\tau_{1}-\gamma_{3} \\
& =\left(\Omega-\bar{\omega}_{1}\right) T_{0}+\tau_{1}-\gamma_{3} \\
\varphi_{2} & =2 \varphi_{1}+\gamma_{1}-\xi_{2} T_{1} \\
& =\left(2 \Omega-\bar{\omega}_{2}\right) T_{0}+2 \tau_{1}+\gamma_{1}-2 \gamma_{3} \\
\varphi_{3} & =\varphi_{1}+\varphi_{1}+\gamma_{2}-\xi_{3} T_{1} \\
& =\left(3 \Omega-\bar{\omega}_{3}\right) T_{0}+3 \tau_{1}+\gamma_{1}+\gamma_{2}-2 \gamma_{3}
\end{aligned}
$$


the response expressed in the original variables can be written as

$$
\begin{aligned}
\eta_{1} & =\epsilon \eta_{11}\left(T_{0}, T_{1}\right)+\epsilon^{2} \eta_{12}\left(T_{0}, T_{1}\right)+\cdots \\
& =\epsilon a_{1} \cos \left(\Omega t+\tau_{1}-\gamma_{3}\right)+O\left(\epsilon^{2}\right) \\
\eta_{2} & =\epsilon \eta_{21}\left(T_{0}, T_{1}\right)+\epsilon^{2} \eta_{22}\left(T_{0}, T_{1}\right)+\cdots \\
& =\epsilon a_{2} \cos \left(2 \Omega t+2 \tau_{1}+\gamma_{1}-2 \gamma_{3}\right)+O\left(\epsilon^{2}\right) \\
\eta_{3} & =\epsilon \eta_{31}\left(T_{0}, T_{1}\right)+\epsilon^{2} \eta_{32}\left(T_{0}, T_{1}\right)+\cdots \\
& =\epsilon a_{3} \cos \left(3 \Omega t+3 \tau_{1}+\gamma_{1}+\gamma_{2}-2 \gamma_{3}\right)+O\left(\epsilon^{2}\right)
\end{aligned}
$$

\section{Simplified model}

From our earlier comments that we are looking for a simplest-possible model, it is interesting to investigate whether Equations (30)-(32) and (40)-(42) can be further simplified. From Figs 3 and 4, we see that $\left|\eta_{1}\right| \gg\left|\eta_{2}\right|$ and $\left|\eta_{1}\right| \gg\left|\eta_{3}\right|$. This means that it is reasonable to assume that the energy transfer into $\eta_{2}$ and $\eta_{3}$ comes directly from $\eta_{1}$. In other words, we consider only the nonlinear terms in Equations (30)-(32) which are nonlinear only with respect to $\eta_{1}$. Using this observation, it means that Equations (30)-(32) can be simplified to

$$
\begin{aligned}
& \ddot{\eta}_{1}+2 \bar{\mu}_{1} \dot{\eta}_{1}+\bar{\omega}_{1}^{2} \eta_{1}=\alpha_{11} \eta_{1}^{3}+F_{1} \cos \left(\Omega t+\tau_{1}\right) \\
& \ddot{\eta}_{2}+2 \bar{\mu}_{2} \dot{\eta}_{2}+\bar{\omega}_{2}^{2} \eta_{2}=\alpha_{24} \eta_{1}^{2} \\
& \ddot{\eta}_{3}+2 \bar{\mu}_{3} \dot{\eta}_{3}+\bar{\omega}_{3}^{2} \eta_{3}=\alpha_{34} \eta_{1}^{3}
\end{aligned}
$$

From these equations, some conclusions can be made directly:

-Equations (112) and (113) consist of two linear oscillators which are forced respectively by the nonlinear terms $\alpha_{24} \eta_{1}^{2}$ and $\alpha_{34} \eta_{1}^{3}$. This means that energy transfer from $\eta_{1}$ to $\eta_{2}$ and $\eta_{3}$ will take place irrespectively of the values of $\bar{\omega}_{2}$ and $\bar{\omega}_{3}$. However, as the perturbation analysis indicated, this energy transfer will be largest when $\bar{\omega}_{2} \approx 2 \bar{\omega}_{1}$ and $\bar{\omega}_{3} \approx 3 \bar{\omega}_{1}$. We also see directly that when the forcing is small (small $F_{1}$ and $\eta_{1}$ ), the excitation of $\eta_{2}$ and $\eta_{3}$ will be small and can be neglected. However when the forcing is large, the response of $\eta_{2}$ and $\eta_{3}$ cannot be neglected. This is in full agreement with the experimental observations.

-By assuming that $\eta_{1}$ has been driven into steady-state, i.e., $\eta_{1}=A_{1} \cos \left(\bar{\omega}_{1} t\right)$, Equations (112) and (113) can be written as

$$
\begin{aligned}
& \ddot{\eta}_{2}+2 \bar{\mu}_{2} \dot{\eta}_{2}+\bar{\omega}_{2}^{2} \eta_{2}=\frac{\alpha_{24} A_{1}^{2}}{2}\left(1+\cos \left(2 \bar{\omega}_{1} t\right)\right) \\
& \ddot{\eta}_{3}+2 \bar{\mu}_{3} \dot{\eta}_{3}+\bar{\omega}_{3}^{2} \eta_{3}=\frac{\alpha_{34} A_{1}^{3}}{4}\left(3 \cos \left(\bar{\omega}_{1} t\right)+\cos \left(3 \bar{\omega}_{1} t\right)\right.
\end{aligned}
$$

This means that when the system is in steady-state, one is able to use a linear observer, e.g. a Kalman filter or a Luenberger observer.

\section{Simulations}

Equations (111)-(113) were simulated using SIMULINK ${ }^{\mathrm{TM}}$. The following numerical parameters were used (note that we scaled down the frequencies by a factor of $\left.10^{4}\right): f_{1}=4, \bar{\mu}_{1}=0.04, \bar{\mu}_{2}=0.0005, \bar{\mu}_{3}=0.5, \bar{\omega}_{1}=9.1[\mathrm{rad} / \mathrm{s}], \bar{\omega}_{2}=38.5[\mathrm{rad} / \mathrm{s}]$, $\bar{\omega}_{3}=27.3[\mathrm{rad} / \mathrm{s}], \alpha_{11}=-0.03, \alpha_{24}=-1$, and $\alpha_{34}=-0.0006$. The result is shown in Fig. 10. 


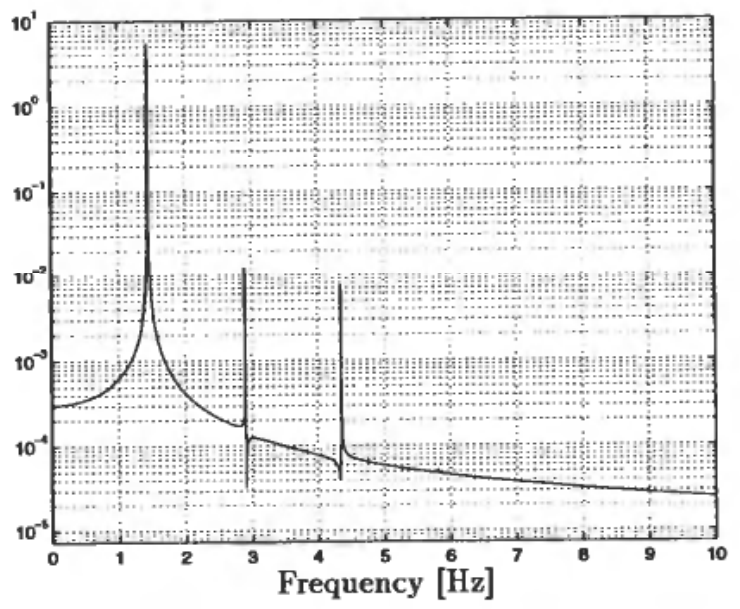

Figure 10. The Fourier spectrum of the steady state response of Equations (111)-(113).

By comparing Fig. 10 with Fig. 4, we see that (111)-(113) are a good description of the experimental results.

\section{Conclusions}

In this paper we have proposed a nonlinear 3-mode coupled model of a cylinder gyroscope made of steel with attached piezoelectric transducers. The model was derived based on experimental results and by using the Donnell-Mushtari theory and Lagrange's equation.

We then analyzed the model using the method of multiple-scales. It was shown analytically that the nonlinearities produced perfect tuning for the primary (external) resonance as well as the internal resonances if the linear eigenfrequencies were commensurable. This is important to avoid when designing gyroscopes.

Since we ultimately were looking for a simple model, we showed how the proposed model could be simplified which is important from a control design point of view. Simulation of the simplified model was provided, and the result corresponded well to the experimental observations. Future research will focus on designing nonlinear observer and vibration damping controller for the gyroscope, which hopefully will enable us to improve the system's performance.

\section{ACKNOWLEDGEMENTS}

This paper has benefited from the reviewers' constructive suggestions and comments.

\section{Appendix A. Derivation of axial mode shape functions}

We will here derive the axial mode shape functions using Hamilton's principle applied to a non-rotating cylinder with no ceramics. The derivation is based on (Burdess 1986; Soedel 1993). Hamilton's principle becomes

$$
\delta \int_{t_{0}}^{t_{1}}(T-U) d t=0
$$


where

$$
T=\frac{1}{2} \rho R h \int_{0}^{l} \int_{0}^{2 \pi}\left(\dot{u}_{1}^{2}+\dot{u}_{2}^{2}+\dot{u}_{3}^{2}\right) d \theta d x
$$

and

$$
U=\frac{1}{2} \int_{0}^{l} \int_{0}^{2 \pi} \int_{-\frac{h}{2}}^{\frac{h}{2}}\left(\sigma_{x x} \varepsilon_{x x}^{l i n}+\sigma_{0 \theta} \varepsilon_{\theta \theta}^{l i n}+\sigma_{x \theta} \varepsilon_{x \theta}^{l i n}+\sigma_{x z} \varepsilon_{x z}^{l i n}+\sigma_{\theta z} \varepsilon_{0 z}^{l i n}\right) R d z d \theta d x
$$

Inspecting each term, we get

$$
\begin{aligned}
\delta \int_{t_{0}}^{t_{1}} T d t & =\delta \int_{t_{0}}^{t_{1}} \frac{1}{2} \rho R h \int_{0}^{t} \int_{0}^{2 \pi}\left(\dot{u}_{1}^{2}+\dot{u}_{2}^{2}+\dot{u}_{3}^{2}\right) d \theta d x d t \\
& =\rho R h \int_{t_{0}}^{t_{1}} \int_{0}^{t} \int_{0}^{2 \pi}\left(\dot{u}_{1} \delta \dot{u}_{1}+\dot{u}_{2} \delta \dot{u}_{2}+\dot{u}_{3} \delta \dot{u}_{3}\right) d \theta d x d t
\end{aligned}
$$

We notice that

$$
\begin{gathered}
\left.\int_{t_{0}}^{t_{1}} \dot{u}_{i} \delta \dot{u}_{i}=\left[\dot{u}_{i} \delta u_{i}\right]\right]_{t_{0}}^{t_{1}}-\int_{t_{0}}^{t_{1}} \ddot{u}_{i} \delta u_{i} d t \\
\Downarrow \\
\int_{t_{0}}^{t_{1}} \dot{u}_{i} \delta \dot{u}_{i}=-\int_{t_{0}}^{t_{1}} \ddot{u}_{i} \delta u_{i} d t
\end{gathered}
$$

Thus

$$
\delta \int_{t_{0}}^{t_{1}} T d=-\rho R h \int_{t_{0}}^{t_{1}} \int_{0}^{l} \int_{0}^{2 \pi}\left(\ddot{u}_{1} \delta u_{1}+\ddot{u}_{2} \delta u_{2}+\ddot{u}_{3} \delta u_{3}\right) d \theta d x d t
$$

Introduce

$$
\begin{aligned}
& \kappa_{1}=-\frac{\partial u_{3}}{\partial x} \\
& \kappa_{2}=-\frac{\partial u_{3}}{\partial \theta}
\end{aligned}
$$

Then using (1)-(2)

$$
\begin{aligned}
& u_{1}^{z}=u_{1}+z \kappa_{1} \\
& u_{2}^{z}=u_{2}+\frac{z}{R} \kappa_{2}
\end{aligned}
$$

and using (12)

$$
\begin{aligned}
& k_{11}=\frac{\partial \kappa_{1}}{\partial x} \\
& k_{22}=\frac{1}{R^{2}} \frac{\partial \kappa_{2}}{\partial \theta} \\
& k_{12}=\frac{2}{R} \frac{\partial \kappa_{2}}{\partial x}
\end{aligned}
$$


From (118), we get

$$
d U=\frac{1}{2}\left(\sigma_{x x} \varepsilon_{x x}^{l i n}+\sigma_{\theta \theta} \varepsilon_{0 \theta}^{l i n}+\sigma_{x \theta} \varepsilon_{x \theta}^{l i n}+\sigma_{x z} \varepsilon_{x z}^{l i n}+\sigma_{0 z} \varepsilon_{0 z}^{l i n}\right) d V
$$

where $d V=R d z d \theta d x$.

We notice that we have to keep the transverse shear terms, even though we have previously assumed $\varepsilon_{x z}$ and $\varepsilon_{0 z}$ to be negligible, to obtain expressions for $\kappa_{1}$ and $\kappa_{2}$ (Soedel 1993).

Let

$$
G=\frac{1}{2}\left(\sigma_{x x} \varepsilon_{x x}^{l i n}+\sigma_{\theta \theta} \varepsilon_{\theta \theta}^{l i n}+\sigma_{x \theta} \varepsilon_{x \theta}^{l i n}+\sigma_{x z} \varepsilon_{x z}^{l i n}+\sigma_{0 z} \varepsilon_{\theta z}^{l i n}\right)
$$

then

$$
\int_{t_{0}}^{t_{1}} \delta U d t=\int_{t_{0}}^{t_{1}} \int_{0}^{t} \int_{0}^{2 \pi} \int_{-\frac{h}{2}}^{\frac{h}{2}} \delta G d V d t
$$

where

$$
\delta G=\frac{\delta G}{\delta \varepsilon_{x x}^{\operatorname{lin}}} \delta \varepsilon_{x x}^{l i n}+\frac{\delta G}{\delta \varepsilon_{\theta \theta}^{\operatorname{lin}}} \varepsilon_{\theta \theta}^{\operatorname{lin}}+\frac{\delta G}{\delta \varepsilon_{x \theta}^{\operatorname{lin}}} \varepsilon_{x \theta}^{l i n}+\frac{\delta G}{\delta \varepsilon_{x z}^{\operatorname{lin}}} \varepsilon_{x z}^{\operatorname{lin}}+\frac{\delta G}{\delta \varepsilon_{\theta z}^{\operatorname{lin}}} \boldsymbol{\varepsilon}_{\theta z}^{\operatorname{lin}}
$$

Using (131), we see that

$$
\frac{\delta G}{\delta \varepsilon_{x x}^{\operatorname{lin}}} \delta \varepsilon_{x x}^{\operatorname{lin}}=\frac{1}{2}\left(\frac{\delta \sigma_{x x}}{\delta \varepsilon_{x x}^{\operatorname{lin}}} \varepsilon_{x x}^{\text {lin }}+\sigma_{x x}+\frac{\partial \sigma_{\theta \theta}}{\partial \varepsilon_{x x}^{l i n}} \varepsilon_{i \theta}^{\operatorname{lin}}\right) \varepsilon_{x x}^{\operatorname{lin}}
$$

Then from (13)

$$
\begin{aligned}
\frac{\partial G}{\partial \varepsilon_{x x}^{\text {lin }}} & =\frac{1}{2}\left(c_{11} \varepsilon_{x x}^{\text {lin }}+\sigma_{x x}+c_{12} \varepsilon_{\theta \theta}^{\text {lin }}\right) \delta \varepsilon_{x x}^{\text {lin }} \\
& =\frac{1}{2}\left(\sigma_{x x}+\sigma_{x x}\right) \delta \varepsilon_{x x}^{l i n} \\
& =\sigma_{x x} \delta \varepsilon_{x x}^{\text {lin }}
\end{aligned}
$$
that

Proceeding with the other terms in (133) in an analog fashion, and using the fact

$$
\begin{aligned}
& \varepsilon_{x x}^{l i n}=\kappa_{1}+\frac{\partial u_{3}}{\partial x} \\
& \varepsilon_{\theta x}^{\operatorname{lin}}=\kappa_{2}+\frac{\partial u_{3}}{\partial \theta}
\end{aligned}
$$

we get

$$
\begin{aligned}
\int_{t_{0}}^{t_{1}} \delta U d t= & \int_{0}^{l} \int_{0}^{2 \pi} \int_{-\frac{h}{2}}^{\frac{h}{2}}\left(\sigma_{x x} \varepsilon_{x x}^{l i n}+\sigma_{\theta \theta} \varepsilon_{\partial \theta}^{l i n}+\sigma_{x \theta} \varepsilon_{x \theta}^{l i n}+\sigma_{x z} \varepsilon_{x z}^{l i n}+\sigma_{\theta z} \varepsilon_{\theta z}^{l i n}\right) R d z d \theta d x d t \\
= & \int_{0}^{l} \int_{0}^{2 \pi} \int_{-\frac{h}{2}}^{\frac{h}{2}}\left[\sigma_{x x} \delta\left(\varepsilon_{11}^{l i n}+z k_{11}\right)+\sigma_{\theta \theta} \delta\left(\varepsilon_{22}^{l i n}+z k_{22}\right)+\sigma_{x \theta} \delta\left(\varepsilon_{12}^{l i n}+z k_{12}\right)\right. \\
& \left.+\sigma_{x z} \delta\left(\kappa_{1}+\frac{\partial u_{3}}{\partial x}\right)+\sigma_{0 z} \delta\left(\kappa_{2}+\frac{\partial u_{3}}{\partial \theta}\right)\right] R d z d \theta d x d t
\end{aligned}
$$




$$
\begin{aligned}
& =\int_{0}^{l} \int_{0}^{2 \pi} \int_{-\frac{h}{2}}^{\frac{h}{2}}\left[\sigma_{x x} \delta\left(\frac{\partial u_{1}}{\partial x}+z \frac{\partial \kappa_{1}}{\partial x}\right)+\sigma_{\theta \theta} \delta\left(\frac{1}{R} \frac{\partial u_{2}}{\partial \theta}+\frac{1}{R} u_{3}+z \frac{1}{R^{2}} \frac{\partial \kappa_{2}}{\partial \theta}\right)\right. \\
& \quad+\sigma_{x \theta} \delta\left(\frac{1}{R} \frac{\partial u_{1}}{\partial \theta}+\frac{\partial u_{2}}{\partial x}+\frac{2}{R} \frac{\partial \kappa_{2}}{\partial x}\right) \\
& \left.\quad+\sigma_{x z} \delta\left(\kappa_{1}+\frac{\partial u_{3}}{\partial x}\right)+\sigma_{0 z} \delta\left(\kappa_{2}+\frac{\partial u_{3}}{\partial \theta}\right)\right] R d z d \theta d x d t \\
& =\int_{0}^{l} \int_{0}^{2 \pi} \int_{-\frac{h}{2}}^{\frac{h}{2}}\left[\sigma_{x x}\left(R \frac{\partial\left(\delta u_{1}\right)}{\partial x}+z R \frac{\partial\left(\delta \kappa_{1}\right)}{\partial x}\right)+\sigma_{\theta \theta}\left(\frac{\partial\left(\delta u_{2}\right)}{\partial \theta}+\delta u_{3}+\frac{z}{R} \frac{\partial\left(\delta \kappa_{2}\right)}{\partial \theta}\right)\right. \\
& \quad+\sigma_{x \theta}\left(\frac{\partial\left(\delta u_{1}\right)}{\partial \theta}+\frac{\partial\left(\delta u_{2}\right)}{\partial x}+2 z \frac{\partial\left(\delta \kappa_{2}\right)}{\partial x}\right) \\
& \left.\quad+\sigma_{x z} R\left(\delta \kappa_{1}+\frac{\partial\left(\delta u_{3}\right)}{\partial x}\right)+\sigma_{\theta z} R\left(\delta \kappa_{2}+\frac{\partial\left(\delta u_{3}\right)}{\partial \theta}\right)\right] d z d \theta d x d t
\end{aligned}
$$

Introducing

$$
\begin{aligned}
& N_{x x}=\int_{-\frac{h}{2}}^{\frac{h}{2}} \sigma_{x x} d z \\
& N_{x \theta}=\int_{-\frac{h}{2}}^{\frac{h}{2}} \sigma_{x \theta} d z \\
& N_{\theta \theta}=\int_{-\frac{h}{2}}^{\frac{h}{2}} \sigma_{\theta \theta} d z \\
& M_{x x}=\int_{-\frac{h}{2}}^{\frac{h}{2}} \sigma_{x x} z d z \\
& M_{\theta \theta}=\int_{-\frac{h}{2}}^{\frac{h}{2}} \sigma_{\theta 0} z d z \\
& M_{x \theta}=\int_{-\frac{h}{2}}^{\frac{h}{2}} \sigma_{x \theta} z d z \\
& Q_{x z}=\int_{-\frac{h}{2}}^{\frac{h}{2}} \sigma_{x z} d z \\
& Q_{\theta z}=\int_{-\frac{h}{2}}^{\frac{h}{2}} \sigma_{\theta z} d z
\end{aligned}
$$

then (138) becomes

$$
\int_{t_{0}}^{t_{1}} \delta U d t=\int_{t_{0}}^{t_{1}} \int_{0}^{l} \int_{0}^{2 \pi}\left[N_{x x} R \frac{\partial\left(\delta u_{1}\right)}{\partial x}+M_{x x} R \frac{\partial\left(\delta \kappa_{1}\right)}{\partial x}+N_{\theta 0}\left(\frac{\partial\left(\delta u_{2}\right)}{\partial \theta}+\delta u_{3}\right)\right.
$$


50

Dag Kristiansen and Olav Egeland

$$
\begin{aligned}
& +M_{\theta \theta} \frac{1}{R} \frac{\partial\left(\delta \kappa_{2}\right)}{\partial \theta}+N_{x \theta}\left(\frac{\partial\left(\delta u_{1}\right)}{\partial \theta}+\frac{\partial\left(\delta u_{2}\right)}{\partial x}\right)+2 M_{x \theta} \frac{\partial\left(\delta \kappa_{2}\right)}{\partial x} \\
& \left.+Q_{x z} R\left(\delta \kappa_{1}+\frac{\partial\left(\delta u_{3}\right)}{\partial x}\right)+Q_{\theta_{z}} R\left(\delta \kappa_{2}+\frac{\partial\left(\delta u_{3}\right)}{\partial \theta}\right)\right]
\end{aligned}
$$

We see that

$$
\begin{gathered}
\int_{0}^{l} \int_{0}^{2 \pi} N_{x x} R \frac{\partial\left(\delta u_{1}\right)}{\partial x} d \theta d x=\int_{0}^{2 \pi} N_{x x} R \delta u_{1} d \theta-R \int_{0}^{l} \int_{0}^{2 \pi} \frac{\partial N_{x x}}{\partial x} \delta u_{1} d \theta d x \\
\int_{0}^{l} \int_{0}^{2 \pi} M_{x x} R \frac{\partial\left(\delta \kappa_{1}\right)}{\partial x} d 0 d x=\int_{0}^{2 \pi} M_{x x} R \delta \kappa_{1} d \theta-R \int_{0}^{l} \int_{0}^{2 \pi} \frac{\partial M_{x x}}{\partial x} \delta \kappa_{1} d \theta d x \\
\int_{0}^{l} \int_{0}^{2 \pi} N_{\theta \theta} \frac{\partial\left(\delta u_{2}\right)}{\partial x} d \theta d x=\int_{0}^{l} N_{\theta \theta} \delta u_{2} d x-\int_{0}^{l} \int_{0}^{2 \pi} \frac{\partial N_{\theta \theta}}{\partial \theta} \delta u_{2} d \theta d x \\
\int_{0}^{l} \int_{0}^{2 \pi} \frac{1}{R} M_{\theta \theta} \frac{\partial\left(\delta \kappa_{2}\right)}{\partial \theta} d 0 d x=\int_{0}^{l} \frac{1}{R} M_{\theta \theta} \delta \kappa_{2} d x-\int_{0}^{l} \int_{0}^{2 \pi} \frac{1}{R} \frac{\partial M_{\theta \theta}}{\partial \theta} \delta \kappa_{2} d \theta d x \\
\int_{0}^{l} \int_{0}^{2 \pi} N_{x \theta} \frac{\partial\left(\delta u_{1}\right)}{\partial \theta} d 0 d x=\int_{0}^{l} N_{x \theta} \delta u_{1} d x-\int_{0}^{l} \int_{0}^{2 \pi} \frac{\partial N_{x \theta}}{\partial \theta} \delta u_{1} d \theta d x \\
\int_{0}^{l} \int_{0}^{2 \pi} N_{x \theta} \frac{\partial\left(\delta u_{2}\right)}{\partial x} d \theta d x=\int_{0}^{2 \pi} N_{x \theta} \delta u_{2} d \theta-\int_{0}^{l} \int_{0}^{2 \pi} \frac{\partial N_{x \theta}}{\partial x} \delta u_{2} d \theta d x \\
2 \int_{0}^{l} \int_{0}^{2 \pi} M_{x \theta} \frac{\partial\left(\delta \kappa_{2}\right)}{\partial x} d \theta d x=2\left[\int_{0}^{2 \pi} M_{x \theta} \delta \kappa_{2} d \theta-\int_{0}^{l} \int_{0}^{2 \pi} \frac{\partial M_{x \theta}}{\partial x} \delta \kappa_{2} d \theta d x\right] \\
\int_{0}^{l} \int_{0}^{2 \pi} R Q_{x z} \frac{\partial\left(\delta u_{3}\right)}{\partial x} d 0 d x=\int_{0}^{2 \pi} R Q_{x z} \delta u_{3} d \theta-\int_{0}^{l} \int_{0}^{2 \pi} R \frac{\partial Q_{x z}}{\partial x} \delta u_{3} d \theta d x \\
\int_{0}^{l} \int_{0}^{2 \pi} R Q_{\theta z} \frac{\partial\left(\delta u_{3}\right)}{\partial \theta} d \theta d x=\int_{0}^{l} R Q_{\theta z} \delta u_{3} d x-\int_{0}^{l} \int_{0}^{2 \pi} R \frac{\partial Q_{\theta z}}{\partial \theta} \delta u_{3} d \theta d x
\end{gathered}
$$

Then

$$
\begin{aligned}
\delta \int_{t_{0}}^{t_{1}} T-U d t= & \int_{t_{0}}^{t_{1}} \int_{0}^{l} \int_{0}^{2 \pi}\left[\left(-\rho R h \ddot{u}_{1}+R \frac{\partial N_{x x}}{\partial x}+\frac{\partial N_{x \theta}}{\partial \theta}\right) \delta u_{1}\right. \\
& +\left(-\rho R h \ddot{u}_{2}+\frac{\partial N_{\theta \theta}}{\partial \theta}+\frac{\partial N_{x \theta}}{\partial x}\right) \delta u_{2} \\
& +\left(-\rho R h \ddot{u}_{3}-N_{\theta \theta}+R \frac{\partial Q_{x z}}{\partial x}+R \frac{Q_{\theta_{z}}}{\partial \theta}\right) \delta u_{3} \\
& +\left(R \frac{\partial M_{x x}}{\partial x}-R Q_{x x}\right) \delta \kappa_{1}
\end{aligned}
$$




$$
\begin{aligned}
& \left.+\left(\frac{1}{R} \frac{\partial M_{\theta \theta}}{\partial \theta}-R Q_{\theta_{z}}+2 \frac{\partial M_{x \theta}}{\partial x}\right) \delta \kappa_{2}\right] d \theta d x d t \\
& -\int_{t_{0}}^{t_{1}} \int_{0}^{t}\left[N_{x \theta} \delta u_{1}+N_{\theta \theta} \delta u_{2}+R Q_{\theta_{z}} \delta u_{3}+\frac{1}{R} M_{\theta \theta} \delta \kappa_{2}\right] d x d t \\
& -\int_{t_{0}}^{t_{1}} \int_{0}^{2 \pi}\left[N_{x x} R \delta u_{1}+N_{x \theta} \delta u_{2}+R Q_{x z} \delta u_{3}+M_{x x} R \delta \kappa_{1}+2 M_{x \theta} \delta \kappa_{2}\right] d \theta d t \\
& =0
\end{aligned}
$$

which gives the following three differential equations

$$
\begin{gathered}
-\rho R h \ddot{u}_{1}+R \frac{\partial N_{x x}}{\partial x}+\frac{\partial N_{x \theta}}{\partial \theta}=0 \\
-\rho R h \ddot{u}_{2}+\frac{\partial N_{\theta \theta}}{\partial \theta}+\frac{\partial N_{x \theta}}{\partial x}=0 \\
-\rho R h \ddot{u}_{3}-N_{\theta \theta}+R \frac{\partial^{2} M_{x x}}{\partial x^{2}}+\frac{1}{R} \frac{\partial^{2} M_{\theta \theta}}{\partial \theta^{2}}+2 \frac{\partial^{2} M_{x \theta}}{\partial x \partial \theta}=0
\end{gathered}
$$

Using (139)-(146), (13), and (5)-(12), we get

$$
\begin{aligned}
& N_{x x}=h\left(c_{11} \frac{\partial u_{1}}{\partial x}+\frac{c_{12}}{R}\left(\frac{\partial u_{2}}{\partial \theta}+u_{3}\right)\right) \\
& N_{\theta \theta}=h\left(c_{12} \frac{\partial u_{1}}{\partial x}+\frac{c_{11}}{R}\left(\frac{\partial u_{2}}{\partial \theta}+u_{3}\right)\right) \\
& N_{x \theta}=c_{33} h\left(\frac{1}{R} \frac{\partial u_{1}}{\partial \theta}+\frac{\partial u_{2}}{\partial x}\right) \\
& M_{x x}=\frac{h^{3}}{12}\left(-c_{11} \frac{\partial^{2} u_{3}}{\partial x^{2}}-\frac{c_{12}}{R^{2}} \frac{\partial^{2} u_{3}}{\partial \theta^{2}}\right) \\
& M_{\theta \theta}=\frac{h^{3}}{12}\left(-c_{12} \frac{\partial^{2} u_{3}}{\partial x^{2}}-\frac{c_{11}}{R^{2}} \frac{\partial^{2} u_{3}}{\partial \theta^{2}}\right) \\
& M_{x \theta}=-\frac{h^{3}}{6 R} \frac{\partial^{2} u_{3}}{\partial x \partial \theta}
\end{aligned}
$$

Thus, (158) can be written

$$
-\rho R \ddot{u}_{1}+R c_{11} \frac{\partial^{2} u_{1}}{\partial x^{2}}+c_{12} \frac{\partial^{2} u_{2}}{\partial x \partial \theta}+c_{12} \frac{\partial u_{3}}{\partial x}+\frac{c_{33}}{R} \frac{\partial^{2} u_{1}}{\partial \theta^{2}}+c_{33} \frac{\partial^{2} u_{2}}{\partial x \partial \theta}=0
$$

Since we are dealing with the linear case, we assume the displacements of the form

$$
\boldsymbol{u}(t, x, \theta)=\bar{\eta}\left(\begin{array}{l}
U_{1 n}(x) \cos n \theta \sin \omega_{n} t \\
U_{2 n}(x) \sin n \theta \sin \omega_{n} t \\
U_{3 n}(x) \cos n \theta \sin \omega_{n} t
\end{array}\right)
$$

Inserting this into (166) gives

$$
\rho R \omega_{n}^{2} U_{1 n}+R c_{11} \frac{d^{2} U_{1 n}}{d x^{2}}+c_{12} \frac{d U_{3 n}}{d x}+n\left(c_{12}+c_{33}\right) \frac{d U_{2 n}}{d x}-n^{2} \frac{c_{33}}{R} U_{1 n}=0
$$


In a similar way (159) can be written as

$$
\begin{gathered}
-\rho R \ddot{u}_{2}+c_{12} \frac{\partial^{2} u_{1}}{\partial x \partial \theta}+\frac{c_{11}}{R} \frac{\partial^{2} u_{2}}{\partial \theta}+\frac{c_{11}}{R} \frac{\partial u_{3}}{\partial \theta}+\frac{c_{33}}{R} \frac{\partial^{2} u_{1}}{\partial x \partial \theta}+c_{33} \frac{\partial^{2} u_{2}}{\partial x^{2}} \\
\Downarrow \\
\rho R \omega_{n}^{2} U_{2 n}+c_{33} \frac{d^{2} U_{2 n}}{d x^{2}}-n^{2} \frac{c_{11}}{R} U_{2 n}-n\left(c_{12}+\frac{c_{33}}{R}\right) \frac{d U_{1 n}}{d x}-n \frac{c_{11}}{R} U_{3 n}=0
\end{gathered}
$$

Finally, Equation (160) can be written as

$$
\begin{gathered}
-\rho R \ddot{u}_{3}-c_{12} \frac{\partial u_{1}}{\partial x}-\frac{c_{11}}{R} \frac{\partial u_{2}}{\partial \theta}-\frac{c_{11}}{R} u_{3}-\frac{h^{2} c_{11} R}{12} \frac{\partial^{4} u_{3}}{\partial x^{4}}-\frac{h^{2}}{3 R}\left(\frac{c_{12}}{3 R}+1\right) \frac{\partial^{3} u_{2}}{\partial \theta \partial x^{2}} \\
-\frac{h^{2} c_{11}}{12 R^{3}} \frac{\partial^{4} u_{3}}{\partial \theta^{4}}=0 \\
\Downarrow R \omega_{n}^{2} U_{3 n}-\frac{h^{2}}{12} c_{11} R \frac{d^{4} U_{3 n}}{d x^{4}}+\frac{h^{2}}{3 R} n^{2}\left(\frac{c_{12}}{3 R}+1\right) \frac{d^{2} U_{3 n}}{d x^{2}}-\frac{c_{11}}{R}\left(1+\frac{h^{2}}{12 R^{2}} n^{4}\right) U_{3 n}-\frac{c_{11}}{R} n U_{2 n} \\
-\frac{c_{12}}{R} \frac{d U_{1 n}}{d x}=0
\end{gathered}
$$

The boundary conditions are given as (clamped end)

$$
U_{1 n}=U_{2 n}=U_{3 n}=\frac{d U_{3 n}}{d x}=0 \quad \text { at } x=0
$$

and (free end)

$$
N_{x x}=N_{x \theta}+\frac{M_{x \theta}}{R}=Q_{x z}+\frac{1}{R} \frac{\partial M_{x \theta}}{\partial \theta}=M_{x x}=0 \quad \text { at } x=l
$$

We see that

$$
\begin{gathered}
N_{x x}=0 \\
\Downarrow \\
R c_{11} \frac{d U_{1 n}}{d x}+n c_{12} U_{2 n}+c_{12} U_{3 n}=0 \\
N_{x \theta}+\frac{M_{x} \theta}{R}=0 \\
\Downarrow \\
\frac{d U_{2 n}}{d x}-n \frac{1}{R} U_{1 n}-\frac{h^{2}}{6 R^{2}} n \frac{d U_{3 n}}{d x}=0 \\
Q_{x x}+\frac{1}{R} \frac{\partial M_{x} \theta}{\partial \theta}=0 \\
\Downarrow
\end{gathered}
$$




$$
\begin{gathered}
-\frac{c_{11}}{2} \frac{d^{3} U_{3 n}}{d x^{3}}+\frac{n^{2}}{R^{2}}\left(\frac{c_{12}}{2}+c_{33}\right) \frac{d U_{3 n}}{d x}=0 \\
M_{x x}=0 \\
\Downarrow \\
c_{11} \frac{d^{2} U_{3 n}}{d x^{2}}-n^{2} \frac{c_{11}}{R^{2}} U_{3 n}=0
\end{gathered}
$$

Defining $\tilde{x}=\frac{x}{R}$, (168), (170), (172), (173), (176), (178), (180), and (182) can be written as

$$
\begin{aligned}
& \rho R^{2} \omega_{n}^{2} U_{1 n}+c_{11} \frac{d^{2} U_{1 n}}{d \bar{x}^{2}}+c_{12} \frac{d U_{3 n}}{d \bar{x}}+n\left(c_{12}+c_{33}\right) \frac{d U_{2 n}}{d \bar{x}}-n^{2} c_{33} U_{1 n}=0 \\
& \rho R^{2} \omega_{n}^{2} U_{2 n}+\frac{c_{33}}{R} \frac{d^{2} U_{2 n}}{d \bar{x}^{2}}-n^{2} c_{11} U_{2 n}-n\left(c_{12}+\frac{c_{33}}{R}\right) \frac{d U_{1 n}}{d \bar{x}}-n c_{11} U_{3 n}=0 \\
& \rho R^{4} \omega_{n}^{2} U_{3 n}-\frac{h^{2}}{12} c_{11} \frac{d^{4} U_{3 n}}{d \bar{x}^{4}}+\frac{h^{2}}{3} n^{2}\left(\frac{c_{12}}{3 R}+1\right) \frac{d^{2} U_{3 n}}{d \bar{x}^{2}}-c_{11} R^{2}\left(1+\frac{h^{2}}{12 R^{2}} n^{4}\right) U_{3 n} \\
& -c_{11} R^{2} n U_{2 n}-c_{12} R \frac{d U_{1 n}}{d \bar{x}}=0 \\
& U_{1 n}=U_{2 n}=U_{3 n}=\frac{d U_{3 n}}{d \bar{x}}=0 \quad \text { at } \bar{x}=0 \\
& c_{11} \frac{d U_{1 n}}{d \tilde{x}}+n c_{12} U_{2 n}+c_{12} U_{3 n}=0 \quad \text { at } \bar{x}=\frac{l}{R} \\
& \frac{d U_{2 n}}{d \bar{x}}-n U_{1 n}-\frac{h^{2}}{6 R^{2}} n \frac{d U_{3 n}}{d \bar{x}}=0 \quad \text { at } \bar{x}=\frac{l}{R} \\
& -\frac{c_{11}}{2} \frac{d^{3} U_{3 n}}{d \bar{x}^{3}}+n^{2}\left(\frac{c_{12}}{2}+c_{33}\right) \frac{d U_{3 n}}{d \bar{x}}=0 \quad \text { at } \bar{x}=\frac{l}{R} \\
& c_{11} \frac{d^{2} U_{3 n}}{d \bar{x}^{2}}-n^{2} c_{11} U_{3 n}=0 \quad \text { at } \bar{x}=\frac{l}{R}
\end{aligned}
$$

The solution of (183)-(185) is of the form

$$
\left.\begin{array}{l}
U_{1 n}=A_{1 n} e^{\lambda \hat{x}} \\
U_{2 n}=A_{2 n} e^{\lambda \bar{x}} \\
U_{3 n}=A_{3 n} e^{\lambda \bar{x}}
\end{array}\right\} \quad U_{m n}=A_{m n} e^{\lambda \bar{x}}
$$

where $\lambda$ and $A_{m n}$ are unknown constants. Inserting (191) into (183) gives

$$
\begin{gathered}
\rho R^{2} \omega_{n}^{2} A_{1 n} e^{\lambda \bar{x}}+c_{11} \lambda^{2} A_{1 n} e^{\lambda \bar{x}}+c_{12} \lambda A_{3 n} e^{i \bar{x}}+n\left(c_{12}+c_{33}\right) \lambda A_{2 n} e^{\lambda \bar{x}}-n^{2} c_{33} A_{1 n} e^{\lambda \bar{x}}=0 \\
\mathbb{I} \\
\left(\rho R^{2} \omega_{n}^{2}+c_{11} \lambda^{2}-n^{2} c_{33}\right) A_{1 n}+n\left(c_{12}+c_{33}\right) \lambda A_{2 n}+c_{12} \lambda A_{3 n}=0
\end{gathered}
$$

Inserting (191) into (184) gives

$$
\rho R^{2} \omega_{n}^{2} A_{2 n} e^{\lambda \bar{x}}+\frac{c_{33}}{R} \lambda^{2} A_{2 n} e^{\lambda \bar{x}}-n^{2} c_{11} A_{2 n} e^{2 \bar{x}}-n\left(c_{12}+\frac{c_{33}}{R}\right) \lambda A_{1 n} e^{2 \bar{x}}-n c_{11} A_{3 n} e^{2 \bar{x}}=0
$$




$$
-n\left(c_{12}+\frac{c_{33}}{R}\right) \lambda A_{1 n}+\left(\rho R^{2} \omega_{n}^{2}+\frac{c_{33}}{R} \lambda^{2}-n^{2} c_{11}\right) A_{2 n}-n c_{11} A_{3 n}=0
$$

Inserting (191) into (185) gives

$$
\begin{gathered}
\rho R^{4} \omega_{n}^{2} A_{3 n} e^{\lambda \bar{x}}-\frac{h^{2}}{12} c_{11} \lambda^{4} A_{3 n} e^{\lambda \bar{x}}+\frac{h^{2}}{3} n^{2}\left(\frac{c_{12}}{3 R}+1\right) \lambda^{2} A_{3 n} e^{\lambda \bar{x}} \\
-c_{11} R^{2}\left(1+\frac{h^{2}}{12 R^{2}} n^{4}\right) A_{3 n} e^{\lambda \dot{x}}-c_{11} R^{2} n A_{2 n} e^{\lambda \bar{x}}-c_{12} R \lambda A_{1 n} e^{\lambda \bar{x}}=0 \\
\Uparrow \\
-c_{12} R \lambda A_{1 n}-c_{11} R^{2} n A_{2 n}+\left(\rho R^{2} \omega_{n}^{2}-\frac{h^{2}}{12} c_{11} \lambda^{4}\right. \\
\left.+\frac{h^{2}}{3} n^{2}\left(\frac{c_{12}}{3 R}+1\right) \lambda^{2}-c_{11} R^{2}\left(1+\frac{h^{2}}{12 R^{2}} n^{4}\right)\right) A_{3 n}=0
\end{gathered}
$$

(193), (195), and (197) give

$$
\begin{gathered}
\left(\begin{array}{ccc}
\rho R^{2} \omega_{n}^{2}+c_{11} \lambda^{2}-n^{2} c_{33} & n\left(c_{12}+c_{33}\right) \lambda & c_{12} \lambda \\
-n\left(c_{12}+\frac{c_{33}}{R}\right) \lambda & \rho R^{2} \omega_{n}^{2}+\frac{c_{33}}{R} \lambda^{2}-n^{2} c_{11} & -n c_{11} \\
-c_{12} R \lambda & -c_{11} R^{2} n & \rho R^{4} \omega_{n}^{2}-\frac{h^{2}}{12} c_{11} \lambda^{4} \\
& & +\frac{h^{2}}{3} n^{2}\left(\frac{c_{12}}{3 R}+1\right) \lambda^{2} \\
& -c_{11} R^{2} \\
A_{2 n} \\
A_{3 n} \\
A_{12} \\
\left(1+\frac{h^{2}}{12 R^{2}} n^{4}\right)
\end{array}\right)=0 \\
\mathbb{1} \\
L\left(\begin{array}{c}
A_{1 n} \\
A_{2 n} \\
A_{3 n}
\end{array}\right)=0
\end{gathered}
$$

(199) has a solution if, and only if

$$
\operatorname{det} L=0
$$

which gives an 8 th order polynom in $\lambda$.

Then by substitution of each $\lambda_{k n}$ into (193), (195), and (197) gives the ratios $B_{1 n}=\frac{A_{1 n}}{A_{3 n}}$ and $B_{2 n}=\frac{A_{2 n}}{A_{3 n}}$. The solutions (191) for each $\lambda_{k n}$ are now superimposed to give

$$
U_{i n}(x)=\sum_{k=1}^{8} a_{i k n} C_{k n} e^{\lambda_{k n} x} \quad i=1, \ldots, 3
$$

where

$$
a_{i k n}=\left\{\begin{array}{cl}
B_{i n}\left(\lambda_{k n}, \omega_{n}\right) & i=1,2 \\
1 & i=3
\end{array}\right.
$$

and $C_{k n}, k=1, \ldots, 6$ are unknown constants. Substitution (201) into the boundary conditions (176), (178), (180), and (182) gives a set of eight linear simultaneous equations of the form 


$$
\sum_{j=1}^{8} P_{i j n} C_{j n}=0 \quad i=1, \ldots, 8
$$

Non-trivial solutions for $C_{j n}$ are possible only if

$$
\operatorname{det} P_{i j n}=0
$$

$\omega_{n}$ and $\lambda_{k n}$ are determined from (200) and (204). Then the ratios $\frac{C_{k n}}{C_{8 n}}$ can be calculated by solving (203).

The functions $U_{i n}(x)$ can now be interpreted as the axial mode shapes and can be written

$$
U_{i n}(x)=\varrho \sum_{k=1}^{8} a_{j k n} b_{k n} e^{\lambda_{k n} x}
$$

where

$$
b_{k n}=\left\{\begin{array}{rl}
\frac{C_{k n}}{c_{8 n}} & k=1, \ldots, 7 \\
1 & k=8
\end{array}\right.
$$

and $\varrho$ is chosen to give $U_{3 n}(l)=1$. The choice of the arbitrary parameter $\varrho$ normalizes the mode shape and allows the quantity $\eta_{n}$ in (27) to be interpreted as the radial displacement at the free end of the cylinder.

\section{Appendix B. Definition of parameters}

$$
\begin{aligned}
& L=\frac{\pi}{2} \rho R h \int_{0}^{l}\left(U_{11}^{2}+U_{21}^{2}+U_{31}^{2}\right) d x \dot{\eta}_{1}^{2}+\frac{\pi}{2} \rho R h \int_{0}^{l}\left(U_{12}^{2}+U_{22}^{2}+U_{32}^{2}\right) d x \dot{\eta}_{2}^{2} \\
& +\frac{\pi}{2} \rho R h \int_{0}^{l}\left(U_{13}^{2}+U_{23}^{2}+U_{33}^{2}\right) d x \dot{\eta}_{3}^{2} \\
& +\frac{\epsilon_{3}^{s}}{2 h_{p}} R l_{p} \psi \sum_{i=1}^{8} V_{i}^{2}-e_{31} R \sin 2 \psi\left(\frac{1}{2} \int_{0}^{l p}\left(\frac{d U_{11}}{d x}\right) d x+\frac{1}{R} \int_{0}^{l p} U_{21} d x\right. \\
& \left.+\frac{1}{2 R} \int_{0}^{l p} U_{31} d x\right) \sum_{i=1}^{4}(-1)^{i+1} V_{2 i-1} \eta_{1} \\
& -e_{31} R \sin 4 \psi\left(\frac{1}{4} \int_{0}^{l p}\left(\frac{d U_{12}}{d x}\right) d x+\frac{1}{R} \int_{0}^{l p} U_{22} d x\right. \\
& \left.+\frac{1}{4 R} \int_{0}^{l p} U_{32} d x\right) \sum_{i=1}^{4}(-1)^{i+1} V_{2 i-1} \eta_{2} \\
& -e_{31} R \sin 6 \psi\left(\frac{1}{6} \int_{0}^{l p}\left(\frac{d U_{13}}{d x}\right) d x+\frac{1}{R} \int_{0}^{l p} U_{23} d x\right. \\
& \left.+\frac{1}{6 R} \int_{0}^{l p} U_{33} d x\right) \sum_{i=1}^{4}(-1)^{i+1} V_{2 i-1} \eta_{3} \\
& -\left[\frac{1}{2} \rho R h \pi\left(\omega_{1}^{2} \int_{0}^{l}\left(U_{11}^{2}+U_{21}^{2}+U_{31}^{2}\right) d x\right)+\frac{e_{31}^{2} R h_{p}^{3} \psi}{6 e_{3}^{s}} \int_{0}^{l p}\left(\frac{d^{2} U_{31}}{d x^{2}}-\frac{4}{R^{2}} U_{31}\right)^{2} d x\right] \eta_{1}^{2} \\
& -\left[\frac{1}{2} \rho R h \pi\left(\omega_{2}^{2} \int_{0}^{l}\left(U_{12}^{2}+U_{22}^{2}+U_{32}^{2}\right) d x\right)\right. \\
& \left.+\frac{e_{31}^{2} R h_{p}^{3} \psi}{24 e_{3}^{s}}(4 \psi+\sin 4 \psi) \int_{0}^{l p}\left(\frac{d^{2} U_{32}}{d x^{2}}-\frac{16}{R^{2}} U_{32}\right)^{2} d x\right] \eta_{2}^{2}
\end{aligned}
$$




$$
\begin{aligned}
& -\left[\frac{1}{2} \rho R h \pi\left(\omega_{3}^{2} \int_{0}^{l}\left(U_{13}^{2}+U_{23}^{2}+U_{33}^{2}\right) d x\right)+\frac{e_{31}^{2} R h_{p}^{3} \psi}{6 e_{3}^{s}} \int_{0}^{l p}\left(\frac{d^{2} U_{33}}{d x^{2}}-\frac{36}{R^{2}} U_{33}\right)^{2} d x\right] \eta_{3}^{2} \\
& -h \pi\left[\frac{3 c_{11} R}{32} \int_{0}^{l}\left(\frac{d U_{31}}{d x}\right)^{4} d x+\frac{3 c_{11}}{2 R^{3}} \int_{0}^{l} U_{31}^{4} d x+\frac{1}{2 R}\left(\frac{c_{12}}{2}+c_{33}\right) \int_{0}^{l} U_{31}^{2}\left(\frac{d U_{31}}{d x}\right)^{2} d x\right] \eta_{1}^{4} \\
& -h \pi\left[\frac{3 c_{11} R}{32} \int_{0}^{l}\left(\frac{d U_{32}}{d x}\right)^{4} d x+\frac{24 c_{11}}{R^{3}} \int_{0}^{l} U_{32}^{4} d x+\frac{1}{R}\left(c_{12}+2 c_{33}\right) \int_{0}^{l} U_{32}^{2}\left(\frac{d U_{32}}{d x}\right)^{2} d x\right] \eta_{2}^{4} \\
& -h \pi\left[\frac{3 c_{11} R}{32} \int_{0}^{l}\left(\frac{d U_{33}}{d x}\right)^{4} d x+\frac{243 c_{11}}{2 R^{3}} \int_{0}^{l} U_{33}^{4} d x+\frac{9 c_{33}}{2 R} \int_{0}^{l} U_{33}^{2}\left(\frac{d U_{33}}{d x}\right)^{2} d x\right] \eta_{3}^{4} \\
& -h \pi\left[\frac{3 c_{11} R}{8} \int_{0}^{l}\left(\frac{d U_{31}}{d x}\right)^{2}\left(\frac{d U_{32}}{d x}\right)^{2} d x+\frac{24 c_{11}}{R^{3}} \int_{0}^{l} U_{31}^{2} U_{32}^{2} d x\right. \\
& \left.+\frac{2}{R}\left(c_{12}+2 c_{33}\right) \int_{0}^{l}\left(\frac{d U_{31}}{d x}\right)^{2} U_{32}^{2} d x+\frac{1}{R}\left(\frac{c_{12}}{2}+c_{33}\right) \int_{0}^{l} U_{31}^{2}\left(\frac{d U_{32}}{d x}\right)^{2} d x\right] \eta_{1}^{2} \eta_{2}^{2} \\
& -h \pi\left[\frac{3 c_{11} R}{8} \int_{0}^{l}\left(\frac{d U_{31}}{d x}\right)^{2}\left(\frac{d U_{33}}{d x}\right)^{2} d x+\frac{54 c_{11}}{R^{3}} \int_{0}^{l} U_{31}^{2} U_{33}^{2} d x\right. \\
& \left.+\frac{9}{R}\left(\frac{c_{12}}{2}+c_{33}\right) \int_{0}^{l}\left(\frac{d U_{31}}{d x}\right)^{2} U_{33}^{2} d x+\frac{1}{R}\left(\frac{c_{12}}{2}+c_{33}\right) \int_{0}^{l} U_{31}^{2}\left(\frac{d U_{33}}{d x}\right)^{2} d x\right] \eta_{1}^{2} \eta_{3}^{2} \\
& -h \pi\left[\frac{3 c_{11} R}{8} \int_{0}^{l}\left(\frac{d U_{32}}{d x}\right)^{2}\left(\frac{d U_{33}}{d x}\right)^{2} d x+\frac{216 c_{11}}{R^{3}} \int_{0}^{l} U_{32}^{2} U_{33}^{2} d x\right. \\
& \left.+\frac{9}{R}\left(\frac{c_{12}}{2}+c_{33}\right) \int_{0}^{l}\left(\frac{d U_{32}}{d x}\right)^{2} U_{33}^{2} d x+\frac{2}{R}\left(c_{12}+2 c_{33}\right) \int_{0}^{l} U_{32}^{2}\left(\frac{d U_{33}}{d x}\right)^{2} d x\right] \eta_{2}^{2} \eta_{3}^{2} \\
& -h \pi\left[\frac{c_{11} R}{8} \int_{0}^{l}\left(\frac{d U_{31}}{d x}\right)^{3}\left(\frac{d U_{33}}{d x}\right) d x-\frac{6 c_{11}}{R^{3}} \int_{0}^{l} U_{31}^{3} U_{33} d x\right. \\
& +\frac{3}{R}\left(\frac{c_{12}}{2}+c_{33}\right) \int_{0}^{l} U_{31}\left(\frac{d U_{31}}{d x}\right)^{2} U_{33} d x-\frac{1}{R}\left(\frac{c_{12}}{2}+c_{33}\right) \int_{0}^{l} U_{31}^{2}\left(\frac{d U_{31}}{d x}\right)\left(\frac{d U_{33}}{d x} d x\right] \eta_{1}^{3} \eta_{3} \\
& -h \pi\left[\frac{c_{11} R}{2} \int_{0}^{l}\left(\left(\frac{d U_{11}}{d x}\right)\left(\frac{d U_{31}}{d x}\right)\left(\frac{d U_{32}}{d x}\right)+\frac{1}{2}\left(\frac{d U_{31}}{d x}\right)^{2}\left(\frac{d U_{12}}{d x}\right)\right) d x\right. \\
& +\frac{c_{12}}{R} \int_{0}^{l}\left(4\left(\frac{d U_{11}}{d x}\right) U_{31} U_{32}-U_{31}^{2}\left(\frac{d U_{12}}{d x}\right)\right) d x \\
& +\frac{c_{12}}{2} \int_{0}^{l}\left(2\left(\frac{d U_{31}}{d x}\right)^{2} U_{22}+\frac{1}{2}\left(\frac{d U_{31}}{d x}\right)^{2} U_{32}+2\left(\frac{d U_{31}}{d x}\right) U_{21}\left(\frac{d U_{32}}{d x}\right)\right. \\
& \left.+\left(\frac{d U_{31}}{d x}\right) U_{31}\left(\frac{d U_{32}}{d x}\right)\right) d x \\
& +\frac{c_{11}}{R^{2}} \int_{0}^{l}\left(8 U_{21} U_{31} U_{32}-4 U_{31}^{2} U_{22}+3 U_{31}^{2} U_{32}\right) d x \\
& +c_{33} \int_{0}^{l}\left(U_{31}\left(\frac{d U_{21}}{d x}\right)\left(\frac{d U_{32}}{d x}\right)-2\left(\frac{d U_{21}}{d x}\right)\left(\frac{d U_{31}}{d x}\right) U_{32}-U_{31}\left(\frac{d U_{31}}{d x}\right)\left(\frac{d U_{22}}{d x}\right)\right. \\
& \left.+\frac{4}{R} U_{11}\left(\frac{d U_{31}}{d x}\right) U_{32}-\frac{2}{R}\left[U_{11} U_{31}\left(\frac{d U_{32}}{d x}\right)+\frac{4}{R}\left(\frac{d U_{31}}{d x}\right) U_{31} U_{12}\right) d x\right] \eta_{1}^{2} \eta_{2} \\
& -h \pi\left[\frac{3 c_{11} R}{8} \int_{0}^{l}\left(\frac{d U_{31}}{d x}\right)\left(\frac{d U_{32}}{d x}\right)^{2}\left(\frac{d U_{33}}{d x}\right) d x+\frac{72 c_{11}}{R^{3}} \int_{0}^{l} U_{31} U_{32}^{2} U_{33} d x\right.
\end{aligned}
$$




$$
\begin{aligned}
& +\frac{1}{R}\left(\frac{c_{12}}{2}+c_{33}\right) \int_{0}^{l}\left(-3 U_{31}\left(\frac{d U_{32}}{d x}\right)^{2} U_{33}+12\left(\frac{d U_{31}}{d x}\right) U_{32}\left(\frac{d U_{32}}{d x}\right) U_{33}\right. \\
& \left.\left.-4\left(\frac{d U_{31}}{d x}\right) U_{32}^{2}\left(\frac{d U_{33}}{d x}\right)+4 U_{31} U_{32}\left(\frac{d U_{32}}{d x}\right)\left(\frac{d U_{33}}{d x}\right)\right) d x\right] \eta_{1} \eta_{2}^{2} \eta_{3} \\
& -h \pi\left[\frac { c _ { 1 1 } R } { 2 } \int _ { 0 } ^ { l } \left(\left(\frac{d U_{11}}{d x}\right)\left(\frac{d U_{32}}{d x}\right)\left(\frac{d U_{33}}{d x}\right)+\left(\frac{d U_{31}}{d x}\right)\left(\frac{d U_{12}}{d x}\right)\left(\frac{d U_{33}}{d x}\right)\right.\right. \\
& \left.+\left(\frac{d U_{31}}{d x}\right)\left(\frac{d U_{32}}{d x}\right)\left(\frac{d U_{13}}{d x}\right)\right) d x \\
& +\frac{2 c_{12}}{R} \int_{0}^{l}\left(6\left(\frac{d U_{11}}{d x}\right) U_{32} U_{33}+3 U_{31}\left(\frac{d U_{12}}{d x}\right) U_{33}-2 U_{31} U_{32}\left(\frac{d U_{13}}{d x}\right)\right) d x \\
& +\frac{c_{12}}{2} \int_{0}^{l}\left(6\left(\frac{d U_{31}}{d x}\right)\left(\frac{d U_{32}}{d x}\right) U_{23}+\left(\frac{d U_{31}}{d x}\right)\left(\frac{d U_{32}}{d x}\right) U_{33}+4\left(\frac{d U_{31}}{d x}\right) U_{22}\left(\frac{d U_{33}}{d x}\right)\right. \\
& \left.+\left(\frac{d U_{31}}{d x}\right) U_{32}\left(\frac{d U_{33}}{d x}\right)+2 U_{21}\left(\frac{d U_{32}}{d x}\right)\left(\frac{d U_{33}}{d x}\right)+U_{31}\left(\frac{d U_{32}}{d x}\right)\left(\frac{d U_{33}}{d x}\right)\right) d x \\
& +\frac{2 c_{11}}{R^{2}} \int_{0}^{l}\left(12 U_{21} U_{32} U_{33}+12 U_{31} U_{22} U_{33}-12 U_{31} U_{32} U_{23}+7 U_{31} U_{32} U_{33}\right) d x \\
& +c_{33} \int_{0}^{l}\left(2\left(\frac{d U_{21}}{d x}\right) U_{32}\left(\frac{d U_{33}}{d x}\right)-3\left(\frac{d U_{21}}{d x}\right)\left(\frac{d U_{32}}{d x}\right) U_{33}-3\left(\frac{d U_{31}}{d x}\right)\left(\frac{d U_{22}}{d x}\right) U_{33}\right. \\
& +U_{31}\left(\frac{d U_{22}}{d x}\right)\left(\frac{d U_{33}}{d x}\right)-2\left(\frac{d U_{31}}{d x}\right) U_{32}\left(\frac{d U_{23}}{d x}\right)-U_{31}\left(\frac{d U_{32}}{d x}\right)\left(\frac{d U_{23}}{d x}\right) \\
& +\frac{6}{R} U_{11}\left(\frac{d U_{32}}{d x}\right) U_{33}-\frac{4}{R} U_{11} U_{32}\left(\frac{d U_{33}}{d x}\right)+\frac{12}{R}\left(\frac{d U_{31}}{d x}\right) U_{12} U_{33}-\frac{4}{R} U_{31} U_{12}\left(\frac{d U_{33}}{d x}\right) \\
& \left.\left.+\frac{12}{R}\left(\frac{d U_{31}}{d x}\right) U_{32} U_{13}+\frac{6}{R} U_{31}\left(\frac{d U_{32}}{d x}\right) U_{13}\right) d x\right] \eta_{1} \eta_{2} \eta_{3} \\
& \bar{\alpha}_{1}=\rho R \int_{0}^{l}\left(U_{11}^{2}+U_{21}^{2}+U_{31}^{2}\right) d x \\
& \bar{\alpha}_{2}=\rho R \int_{0}^{l}\left(U_{12}^{2}+U_{22}^{2}+U_{32}^{2}\right) d x \\
& \bar{\alpha}_{3}=\rho R \int_{0}^{l}\left(U_{13}^{2}+U_{23}^{2}+U_{33}^{2}\right) d x \\
& \alpha_{11}=-\frac{1}{\bar{\alpha}_{1}}\left[\frac{3 c_{11} R}{8} \int_{0}^{l}\left(\frac{d U_{31}}{d x}\right)^{4} d x+\frac{6 c_{11}}{R^{3}} \int_{0}^{l} U_{31}^{4} d x+\frac{2}{R}\left(\frac{c_{12}}{2}+c_{33}\right) \int_{0}^{l} U_{31}^{2}\left(\frac{d U_{31}}{d x}\right)^{2} d x\right] \\
& \alpha_{12}=-\frac{1}{\bar{\alpha}_{1}}\left[\frac{3 c_{11} R}{4} \int_{0}^{l}\left(\frac{d U_{31}}{d x}\right)^{2}\left(\frac{d U_{32}}{d x}\right)^{2} d x+\frac{48 c_{11}}{R^{3}} \int_{0}^{l} U_{31}^{2} U_{32}^{2} d x\right. \\
& \left.+\frac{4}{R}\left(c_{12}+2 c_{33}\right) \int_{0}^{l}\left(\frac{d U_{31}}{d x}\right)^{2} U_{32}^{2} d x+\frac{2}{R}\left(\frac{c_{12}}{2}+c_{33}\right) \int_{0}^{l} U_{31}^{2}\left(\frac{d U_{32}}{d x}\right)^{2} d x\right] \\
& \alpha_{13}=-\frac{1}{\bar{\alpha}_{1}}\left[\frac{3 c_{11} R}{4} \int_{0}^{l}\left(\frac{d U_{31}}{d x}\right)^{2}\left(\frac{d U_{33}}{d x}\right)^{2} d x+\frac{108 c_{11}}{R^{3}} \int_{0}^{l} U_{31}^{2} U_{33}^{2} d x\right.
\end{aligned}
$$




$$
\begin{aligned}
& \left.+\frac{18}{R}\left(\frac{c_{12}}{2}+c_{33}\right) \int_{0}^{l}\left(\frac{d U_{31}}{d x}\right)^{2} U_{33}^{2} d x+\frac{2}{R}\left(\frac{c_{12}}{2}+c_{33}\right) \int_{0}^{l} U_{31}^{2}\left(\frac{d U_{33}}{d x}\right)^{2} d x\right] \\
& \alpha_{14}=-\frac{1}{\bar{\alpha}_{1}}\left[\frac{3 c_{11} R}{8} \int_{0}^{l}\left(\frac{d U_{31}}{d x}\right)^{3}\left(\frac{d U_{33}}{d x}\right) d x-\frac{18 c_{11}}{R^{3}} \int_{0}^{l} U_{31}^{2} U_{33} d x\right. \\
& +\frac{9}{R}\left(\frac{c_{12}}{2}+c_{33}\right) \int_{0}^{l} U_{31}\left(\frac{d U_{31}}{d x}\right)^{2} U_{33}^{2} d x \\
& \left.-\frac{3}{R}\left(\frac{c_{12}}{2}+c_{33}\right) \int_{0}^{l} U_{31}^{2}\left(\frac{d U_{31}}{d x}\right)\left(\frac{d U_{33}}{d x}\right) d x\right] \\
& \alpha_{15}=-\frac{2}{\bar{\alpha}_{1}}\left[\frac{c_{11} R}{2} \int_{0}^{l}\left(\left(\frac{d U_{11}}{d x}\right)\left(\frac{d U_{31}}{d x}\right)\left(\frac{d U_{32}}{d x}\right)+\frac{1}{2}\left(\frac{d U_{31}}{d x}\right)\left(\frac{d U_{12}}{d x}\right)\right) d x\right. \\
& +\frac{c_{12}}{R} \int_{0}^{i}\left(4\left(\frac{d U_{11}}{d x}\right)-U_{31} U_{32}-U_{31}^{2}\left(\frac{d U_{12}}{d x}\right)\right) d x \\
& +\frac{c_{12}}{2} \int_{0}^{l}\left(2\left(\frac{d U_{31}}{d x}\right)^{2} U_{22}+\frac{1}{2}\left(\frac{d U_{31}}{d x}\right)^{2} U_{32}+2\left(\frac{d U_{31}}{d x}\right) U_{21}\left(\frac{d U_{32}}{d x}\right)\right. \\
& \left.+\left(\frac{d U_{31}}{d x}\right) U_{31}\left(\frac{d U_{32}}{d x}\right)\right) d x+\frac{c_{11}}{R^{2}} \int_{0}^{l}\left(8 U_{21} U_{31} U_{32}-4 U_{31}^{2} U_{22}+3 U_{31}^{2} U_{32}\right) d x \\
& +c_{33} \int_{0}^{l}\left(U_{31}\left(\frac{d U_{21}}{d x}\right)\left(\frac{d U_{32}}{d x}\right)-2\left(\frac{d U_{21}}{d x}\right)\left(\frac{d U_{31}}{d x}\right) U_{32}-U_{31}\left(\frac{d U_{31}}{d x}\right)\left(\frac{d U_{22}}{d x}\right)\right. \\
& \left.\left.+\frac{4}{R} U_{11}\left(\frac{d U_{31}}{d x}\right) U_{32}-\frac{2}{R} U_{11} U_{31}\left(\frac{d U_{32}}{d x}\right)+\frac{4}{R}\left(\frac{d U_{31}}{d x}\right) U_{31} U_{12}\right) d x\right] \\
& \alpha_{16}=-\frac{1}{\bar{\alpha}_{1}}\left[\frac{3 c_{11} R}{8} \int_{0}^{l}\left(\frac{d U_{31}}{d x}\right)\left(\frac{d U_{32}}{d x}\right)^{2}\left(\frac{d U_{33}}{d x}\right) d x+\frac{72 c_{11}}{R^{3}} \int_{0}^{l} U_{31} U_{32}^{2} U_{33} d x\right. \\
& +\frac{1}{R}\left(\frac{c_{12}}{2}+c_{33}\right) \int_{0}^{l}\left(-3 U_{31}\left(\frac{d U_{32}}{d x}\right)^{2} U_{33}\right. \\
& +12\left(\frac{d U_{31}}{d x}\right) U_{32}\left(\frac{d U_{32}}{d x}\right) U_{33}-4\left(\frac{d U_{31}}{d x}\right) U_{32}^{2}\left(\frac{d U_{33}}{d x}\right) \\
& \left.\left.+4 U_{31} U_{32}\left(\frac{d U_{32}}{d x}\right)\left(\frac{d U_{33}}{d x}\right)\right) d x\right] \\
& \alpha_{17}=-\frac{1}{\bar{\alpha}_{1}}\left[\frac { c _ { 1 1 } R } { 2 } \int _ { 0 } ^ { l } \left(\left(\frac{d U_{11}}{d x}\right)\left(\frac{d U_{32}}{d x}\right)\left(\frac{d U_{33}}{d x}\right)+\left(\frac{d U_{31}}{d x}\right)\left(\frac{d U_{12}}{d x}\right)\left(\frac{d U_{33}}{d x}\right)\right.\right. \\
& \left.+\left(\frac{d U_{31}}{d x}\right)\left(\frac{d U_{32}}{d x}\right)\left(\frac{d U_{13}}{d x}\right)\right) d x+\frac{2 c_{12}}{R} \int_{0}^{l}\left(6\left(\frac{d U_{11}}{d x}\right) U_{32} U_{33}\right. \\
& \left.+3 U_{31}\left(\frac{d U_{12}}{d x}\right) U_{33}-2 U_{31} U_{32}\left(\frac{d U_{13}}{d x}\right)\right) d x+\frac{c_{12}}{2} \int_{0}^{l}\left(6\left(\frac{d U_{31}}{d x}\right)\left(\frac{d U_{32}}{d x}\right) U_{23}\right. \\
& +\left(\frac{d U_{31}}{d x}\right)\left(\frac{d U_{32}}{d x}\right) U_{33}+4\left(\frac{d U_{31}}{d x}\right) U_{22}\left(\frac{d U_{33}}{d x}\right) \\
& \left.+\left(\frac{d U_{31}}{d x}\right) U_{32}\left(\frac{d U_{33}}{d x}\right)+2 U_{21}\left(\frac{d U_{32}}{d x}\right)\left(\frac{d U_{33}}{d x}\right)+U_{31}\left(\frac{d U_{32}}{d x}\right)\left(\frac{d U_{33}}{d x}\right)\right) d x \\
& +\frac{2 c_{11}}{R^{2}} \int_{0}^{l}\left(12 U_{21} U_{32} U_{33}+12 U_{31} U_{22} U_{33}-12 U_{31} U_{32} U_{23}+7 U_{31} U_{32} U_{33}\right) d x
\end{aligned}
$$




$$
\begin{aligned}
& +c_{33} \int_{0}^{l}\left(2\left(\frac{d U_{21}}{d x}\right) U_{32}\left(\frac{d U_{33}}{d x}\right)-3\left(\frac{d U_{21}}{d x}\right)\left(\frac{d U_{32}}{d x}\right) U_{33}-3\left(\frac{d U_{31}}{d x}\right)\left(\frac{d U_{22}}{d x}\right) U_{33}\right. \\
& +U_{31}\left(\frac{d U_{22}}{d x}\right)\left(\frac{d U_{33}}{d x}\right)-2\left(\frac{d U_{31}}{d x}\right) U_{32}\left(\frac{d U_{23}}{d x}\right)-U_{31}\left(\frac{d U_{32}}{d x}\right)\left(\frac{d U_{23}}{d x}\right) \\
& +\frac{6}{R} U_{11}\left(\frac{d U_{32}}{d x}\right) U_{33}-\frac{4}{R} U_{11} U_{32}\left(\frac{d U_{33}}{d x}\right)+\frac{12}{R}\left(\frac{d U_{31}}{d x}\right) U_{12} U_{33}-\frac{4}{R} U_{31} U_{12}\left(\frac{d U_{33}}{d x}\right) \\
& \left.\left.+\frac{12}{R}\left(\frac{d U_{31}}{d x}\right) U_{32} U_{13}+\frac{6}{R} U_{31}\left(\frac{d U_{32}}{d x}\right) U_{13}\right) d x\right] \\
& \alpha_{21}=-\frac{4}{\bar{\alpha}_{2}}\left[\frac{3 c_{11} R}{32} \int_{0}^{l}\left(\frac{d U_{32}}{d x}\right)^{4} d x+\frac{24 c_{11}}{R^{3}} \int_{0}^{l} U_{32}^{4} d x\right. \\
& \left.+\frac{1}{R}\left(c_{12}+2 c_{33}\right) \int_{0}^{l} U_{32}^{2}\left(\frac{d U_{32}}{d x}\right)^{2} d x\right] \\
& \alpha_{22}=-\frac{2}{\bar{\alpha}_{2}}\left[\frac{3 c_{11} R}{8} \int_{0}^{l}\left(\frac{d U_{31}}{d x}\right)^{2}\left(\frac{d U_{32}}{d x}\right)^{2} d x+\frac{24 c_{11}}{R^{3}} \int_{0}^{l} U_{31}^{2} U_{32}^{2} d x\right. \\
& \left.+\frac{2}{R}\left(c_{12}+2 c_{33}\right) \int_{0}^{l}\left(\frac{d U_{31}}{d x}\right)^{2} U_{32}^{2} d x+\frac{1}{R}\left(\frac{c_{12}}{2}+c_{33}\right) \int_{0}^{l} U_{31}^{2}\left(\frac{d U_{32}}{d x}\right)^{2} d x\right] \\
& \alpha_{23}=-\frac{2}{\bar{\alpha}_{2}}\left[\frac{3 c_{11} R}{8} \int_{0}^{l}\left(\frac{d U_{32}}{d x}\right)^{2}\left(\frac{d U_{33}}{d x}\right)^{2} d x+\frac{216 c_{11}}{R^{3}} \int_{0}^{l} U_{32}^{2} U_{33}^{2} d x\right. \\
& \left.+\frac{9}{R}\left(\frac{c_{12}}{2}+c_{33}\right) \int_{0}^{l}\left(\frac{d U_{32}}{d x}\right)^{2} U_{33}^{2} d x+\frac{2}{R}\left(c_{12}+2 c_{33}\right) \int_{0}^{l} U_{32}^{2}\left(\frac{d U_{33}}{d x}\right)^{2} d x\right] \\
& \alpha_{24}=-\frac{1}{\bar{\alpha}_{2}}\left[\frac{c_{11} R}{2} \int_{0}^{l}\left(\left(\frac{d U_{11}}{d x}\right)\left(\frac{d U_{31}}{d x}\right)\left(\frac{d U_{32}}{d x}\right)+\frac{1}{2}\left(\frac{d U_{31}}{d x}\right)^{2}\left(\frac{d U_{12}}{d x}\right)\right) d x\right. \\
& +\frac{c_{12}}{R} \int_{0}^{l}\left(4\left(\frac{d U_{11}}{d x}\right) U_{31} U_{32}-U_{31}^{2}\left(\frac{d U_{12}}{d x}\right)\right) d x \\
& +\frac{c_{12}}{2} \int_{0}^{l}\left(2\left(\frac{d U_{31}}{d x}\right)^{2} U_{22}+\frac{1}{2}\left(\frac{d U_{31}}{d x}\right)^{2} U_{32}\right. \\
& \left.+2\left(\frac{d U_{31}}{d x}\right) U_{21}\left(\frac{d U_{32}}{d x}\right)+\left(\frac{d U_{31}}{d x}\right) U_{31}\left(\frac{d U_{32}}{d x}\right)\right) d x \\
& +\frac{c_{11}}{R^{2}} \int_{0}^{l}\left(8 U_{21} U_{31} U_{32}-4 U_{31}^{2} U_{22}+3 U_{31}^{2} U_{32}\right) d x \\
& +c_{33} \int_{0}^{l}\left(U_{31}\left(\frac{d U_{21}}{d x}\right)\left(\frac{d U_{32}}{d x}\right)-2\left(\frac{d U_{21}}{d x}\right)\left(\frac{d U_{31}}{d x}\right) U_{32}-U_{31}\left(\frac{d U_{31}}{d x}\right)\left(\frac{d U_{22}}{d x}\right)\right. \\
& \left.\left.+\frac{4}{R} U_{11}\left(\frac{d U_{31}}{d x}\right) U_{32}-\frac{2}{R} U_{11} U_{31}\left(\frac{d U_{32}}{d x}\right)+\frac{4}{R}\left(\frac{d U_{31}}{d x}\right) U_{31} U_{12}\right) d x\right] \\
& \alpha_{25}=-\frac{2}{\bar{\alpha}_{2}}\left[\frac{3 c_{11} R}{8} \int_{0}^{l}\left(\frac{d U_{31}}{d x}\right)\left(\frac{d U_{32}}{d x}\right)^{2}\left(\frac{d U_{33}}{d x}\right) d x+\frac{72 c_{11}}{R^{3}} \int_{0}^{l} U_{31} U_{32}^{2} U_{33} d x\right. \\
& +\frac{1}{R}\left(\frac{c_{12}}{2}+c_{33}\right) \int_{0}^{l}\left(-3 U_{31}\left(\frac{d U_{32}}{d x}\right)^{2} U_{33}\right. \\
& +12\left(\frac{d U_{31}}{d x}\right) U_{32}\left(\frac{d U_{32}}{d x}\right) U_{33}-4\left(\frac{d U_{31}}{d x}\right) U_{32}^{2}\left(\frac{d U_{33}}{d x}\right)
\end{aligned}
$$




$$
\begin{aligned}
& \left.\left.+4 U_{31}^{2} U_{32}\left(\frac{d U_{32}}{d x}\right)\left(\frac{d U_{33}}{d x}\right)\right) d x\right] \\
& \alpha_{26}=-\frac{1}{\bar{\alpha}_{2}}\left[\frac { c _ { 1 1 } R } { 2 } \int _ { 0 } ^ { l } \left(\left(\frac{d U_{11}}{d x}\right)\left(\frac{d U_{32}}{d x}\right)\left(\frac{d U_{33}}{d x}\right)+\left(\frac{d U_{31}}{d x}\right)\left(\frac{d U_{12}}{d x}\right)\left(\frac{d U_{33}}{d x}\right)\right.\right. \\
& \left.+\left(\frac{d U_{31}}{d x}\right)\left(\frac{d U_{32}}{d x}\right)\left(\frac{d U_{13}}{d x}\right)\right) d x+\frac{2 c_{12}}{R} \int_{0}^{l}\left(6\left(\frac{d U_{11}}{d x}\right) U_{32} U_{33}\right. \\
& \left.+3 U_{31}\left(\frac{d U_{12}}{d x}\right) U_{33}-2 U_{31} U_{32}\left(\frac{d U_{13}}{d x}\right)\right) d x+\frac{c_{12}}{2} \int_{0}^{l}\left(6\left(\frac{d U_{31}}{d x}\right)\left(\frac{d U_{32}}{d x}\right) U_{23}\right. \\
& +\left(\frac{d U_{31}}{d x}\right)\left(\frac{d U_{32}}{d x}\right) U_{33}+4\left(\frac{d U_{31}}{d x}\right) U_{22}\left(\frac{d U_{33}}{d x}\right) \\
& \left.+\left(\frac{d U_{31}}{d x}\right) U_{32}\left(\frac{d U_{33}}{d x}\right)+2 U_{21}\left(\frac{d U_{32}}{d x}\right)\left(\frac{d U_{33}}{d x}\right)+U_{31}\left(\frac{d U_{32}}{d x}\right)\left(\frac{d U_{33}}{d x}\right)\right) d x \\
& +\frac{2 c_{11}}{R^{2}} \int_{0}^{l}\left(12 U_{21} U_{32} U_{33}+12 U_{31} U_{22} U_{33}-12 U_{31} U_{32} U_{23}+7 U_{31} U_{32} U_{33}\right) d x \\
& +c_{33} \int_{0}^{l}\left(2\left(\frac{d U_{21}}{d x}\right) U_{32}\left(\frac{d U_{33}}{d x}\right)-3\left(\frac{d U_{21}}{d x}\right)\left(\frac{d U_{32}}{d x}\right) U_{33}-3\left(\frac{d U_{31}}{d x}\right)\left(\frac{d U_{22}}{d x}\right) U_{33}\right. \\
& +U_{31}\left(\frac{d U_{22}}{d x}\right)\left(\frac{d U_{33}}{d x}\right)-2\left(\frac{d U_{31}}{d x}\right) U_{32}\left(\frac{d U_{23}}{d x}\right)-U_{31}\left(\frac{d U_{32}}{d x}\right)\left(\frac{d U_{23}}{d x}\right) \\
& +\frac{6}{R} U_{11}\left(\frac{d U_{32}}{d x}\right) U_{33}-\frac{4}{R} U_{11} U_{32}\left(\frac{d U_{33}}{d x}\right)+\frac{12}{R}\left(\frac{d U_{31}}{d x}\right) U_{12} U_{33}-\frac{4}{R} U_{31} U_{12}\left(\frac{d U_{33}}{d x}\right) \\
& \left.\left.+\frac{12}{R}\left(\frac{d U_{31}}{d x}\right) U_{32} U_{13}+\frac{6}{R} U_{31}\left(\frac{d U_{32}}{d x}\right) U_{13}\right) d x\right] \\
& \alpha_{31}=-\frac{4}{\bar{\alpha}_{3}}\left[\frac{3 c_{11} R}{32} \int_{0}^{l}\left(\frac{d U_{33}}{d x}\right)^{4} d x+\frac{243 c_{11}}{2 R^{3}} \int_{0}^{l} U_{33}^{4} d x+\frac{9 c_{33}}{2 R} \int_{0}^{l} U_{33}^{2}\left(\frac{d U_{33}}{d x}\right)^{2} d x\right] \\
& \alpha_{32}=-\frac{2}{\bar{\alpha}_{3}}\left[\frac{3 c_{11} R}{8} \int_{0}^{l}\left(\frac{d U_{31}}{d x}\right)^{2}\left(\frac{d U_{33}}{d x}\right)^{2} d x+\frac{54 c_{11}}{R^{3}} \int_{0}^{l} U_{31}^{2} U_{33}^{2} d x\right. \\
& \left.+\frac{9}{R}\left(\frac{c_{12}}{2}+c_{33}\right) \int_{0}^{l}\left(\frac{d U_{31}}{d x}\right)^{2} U_{33}^{2} d x+\frac{1}{R}\left(\frac{c_{12}}{2}+c_{33}\right) \int_{0}^{l} U_{31}^{2}\left(\frac{d U_{33}}{d x}\right)^{2} d x\right] \\
& \alpha_{33}=-\frac{2}{\bar{\alpha}_{3}}\left[\frac{3 c_{11} R}{8} \int_{0}^{l}\left(\frac{d U_{32}}{d x}\right)^{2}\left(\frac{d U_{33}}{d x}\right)^{2} d x+\frac{216 c_{11}}{R^{3}} \int_{0}^{l} U_{32}^{2} U_{33}^{2} d x\right. \\
& \left.+\frac{9}{R}\left(\frac{c_{12}}{2}+c_{33}\right) \int_{0}^{l}\left(\frac{d U_{32}}{d x}\right)^{2} U_{33}^{2} d x+\frac{2}{R}\left(c_{12}+2 c_{33}\right) \int_{0}^{l} U_{32}^{2}\left(\frac{d U_{33}}{d x}\right)^{2} d x\right] \\
& \alpha_{34}=-\frac{1}{\bar{\alpha}_{3}}\left[\frac{3 c_{11} R}{8} \int_{0}^{l}\left(\frac{d U_{31}}{d x}\right)^{3}\left(\frac{d U_{33}}{d x}\right) d x-\frac{6 c_{11}}{R^{3}} \int_{0}^{l} U_{31}^{3} U_{33} d x\right. \\
& \left.+\frac{3}{R}\left(\frac{c_{12}}{2}+c_{33}\right) \int_{0}^{l} U_{31}\left(\frac{d U_{31}}{d x}\right)^{2} U_{33} d x-\frac{1}{R}\left(\frac{c_{12}}{2}+c_{33}\right) \int_{0}^{l} U_{31}^{2}\left(\frac{d U_{31}}{d x}\right)\left(\frac{d U_{33}}{d x}\right) d x\right]
\end{aligned}
$$

$\alpha_{35}=-\frac{1}{\bar{\alpha}_{3}}\left[\frac{3 c_{11} R}{8} \int_{0}^{l}\left(\frac{d U_{31}}{d x}\right)\left(\frac{d U_{32}}{d x}\right)^{2}\left(\frac{d U_{33}}{d x}\right) d x+\frac{72 c_{11}}{R^{3}} \int_{0}^{l} U_{31} U_{32}^{2} U_{33} d x\right.$ 


$$
\begin{aligned}
+ & \frac{1}{R}\left(\frac{c_{12}}{2}+c_{33}\right) \int_{0}^{l}\left(-3 U_{31}\left(\frac{d U_{32}}{d x}\right)^{2} U_{33}\right. \\
+ & 12\left(\frac{d U_{31}}{d x}\right) U_{32}\left(\frac{d U_{32}}{d x}\right) U_{33}-4\left(\frac{d U_{31}}{d x}\right) U_{32}^{2}\left(\frac{d U_{33}}{d x}\right) \\
& \left.\left.+4 U_{31}^{2} U_{32}\left(\frac{d U_{32}}{d x}\right)\left(\frac{d U_{33}}{d x}\right)\right) d x\right] \\
\alpha_{36}= & -\frac{1}{\bar{\alpha}_{3}}\left[\frac { c _ { 1 1 } R } { 2 } \int _ { 0 } ^ { l } \left(\left(\frac{d U_{11}}{d x}\right)\left(\frac{d U_{32}}{d x}\right)\left(\frac{d U_{33}}{d x}\right)+\left(\frac{d U_{31}}{d x}\right)\left(\frac{d U_{12}}{d x}\right)\left(\frac{d U_{33}}{d x}\right)\right.\right. \\
& \left.+\left(\frac{d U_{31}}{d x}\right)\left(\frac{d U_{32}}{d x}\right)\left(\frac{d U_{13}}{d x}\right)\right) d x+\frac{2 c_{12}}{R} \int_{0}^{l}\left(6\left(\frac{d U_{11}}{d x}\right) U_{32} U_{33}\right. \\
& \left.+3 U_{31}\left(\frac{d U_{12}}{d x}\right) U_{33}-2 U_{31} U_{32}\left(\frac{d U_{13}}{d x}\right)\right) d x+\frac{c_{12}}{2} \int_{0}^{l}\left(6\left(\frac{d U_{31}}{d x}\right)\left(\frac{d U_{32}}{d x}\right) U_{23}\right. \\
& +\left(\frac{d U_{31}}{d x}\right)\left(\frac{d U_{32}}{d x}\right) U_{33}+4\left(\frac{d U_{31}}{d x}\right) U_{22}\left(\frac{d U_{33}}{d x}\right) \\
& \left.+\left(\frac{d U_{31}}{d x}\right) U_{32}\left(\frac{d U_{33}}{d x}\right)+2 U_{21}\left(\frac{d U_{32}}{d x}\right)\left(\frac{d U_{33}}{d x}\right)+U_{31}\left(\frac{d U_{32}}{d x}\right)\left(\frac{d U_{33}}{d x}\right)\right) d x \\
& +\frac{2 c_{11}}{R^{2}} \int_{0}^{l}\left(12 U_{21} U_{32} U_{33}+12 U_{31} U_{22} U_{33}-12 U_{31} U_{32} U_{23}+7 U_{31} U_{32} U_{33}\right) d x \\
& +c_{33} \int_{0}^{l}\left(2\left(\frac{d U_{21}}{d x}\right) U_{32}\left(\frac{d U_{33}}{d x}\right)-3\left(\frac{d U_{21}}{d x}\right)\left(\frac{d U_{32}}{d x}\right) U_{33}-3\left(\frac{d U_{31}}{d x}\right)\left(\frac{d U_{22}}{d x}\right) U_{33}\right. \\
+ & U_{31}\left(\frac{d U_{22}}{d x}\right)\left(\frac{d U_{33}}{d x}\right)-2\left(\frac{d U_{31}}{d x}\right) U_{32}\left(\frac{d U_{23}}{d x}\right)-U_{31}\left(\frac{d U_{32}}{d x}\right)\left(\frac{d U_{23}}{d x}\right) \\
+ & \frac{6}{R} U_{11}\left(\frac{d U_{32}}{d x}\right) U_{33}-\frac{4}{R} U_{11} U_{32}\left(\frac{d U_{33}}{d x}\right)+\frac{12}{R}\left(\frac{d U_{31}}{d x}\right) U_{12} U_{33}-\frac{4}{R} U_{31} U_{12}\left(\frac{d U_{33}}{d x}\right) \\
+ & \left.\left.\frac{12}{R}\left(\frac{d U_{31}}{d x}\right) U_{32} U_{13}+\frac{6}{R} U_{31}\left(\frac{d U_{32}}{d x}\right) U_{13}\right) d x\right]
\end{aligned}
$$

REFERENCES

ABE, H., Yoshida, T., Kobayashi, H. and NAKAmURA, K. (1996). Temperature Dependence of Zero Offset of Piezoelectric-Ceramic Cylinder Vibratory Gyroscope. Japanese Journal of Applied Physics, 35, pp. 5031-5033.

ABE, H., Yoshida, T. and NaKamura, K. (1996). Vibration Mode Analysis of PiezoelectricCeramic Cylinder Vibratory Gyroscopes with Interdigital Electrodes. Electronics and Communications in Japan, 79, pp. 53-60.

ABE, H., YoshidA, T. and TuRugA, K. (1992). Piezoelectric-Ceramic Cylinder Vibratory Gyroscope. Japanese Journal of Applied Physics, 31, pp. 3061-3063.

ANDERS, J. and PEARSON, R. (1994). Applications of the 'START' Vibratory Gyroscope. GEC Review, 9, pp. 168-175.

BLEVINS, R. (1979). Formulas for Natural Frequency and Mode Shapes, Van Nostrand Reinhold, New York.

Burdess, J. (1986). The dynamics of a thin piezoelectric cylinder gyroscope. Proceedings of Institute of Mechanical Engineers, 200, pp. 271-280.

CHEN, J. and BABCOCK, C. (1975). Nonlinear Vibration of Cylindrical Shells. AIAA Journal, 13, pp. 868-876.

CHU, H. (1961). Influence of Large Amplitudes on Flexural Vibrations of a Thin Circular Cylindrical Shell. Journal of Aerospace Sciences, 28, pp. 602-609. 
Evan-Iwanowski, R. (1976). Resonance Oscillations in Mechanical Systems, Elsevier, New York.

Fox, C. (1984). Vibratory gyroscopic sensors, in DGON Symposium on Gyro Technology, Stuttgart, Germany.

Fox, C. (1988). Vibrating Cylinder Rate Gyro, Theory of Operation and Error Analysis, in DGON Symposium on Gyro Technology, Stuttgart, Germany.

Hagood, N. Chung, W. and von Flowtow, A. (1990). Modelling of Piezoelectric Actuator Dynamics for Active Structural Control. Journal of Intelligent Material, Systems and Structures, 1, pp. 327-354.

Kagawa, Y., Tsuchiya, T. and Kawashima, T. (1996). Finite Element Simulation of Piezoelectric Vibrator Gyroscopes. IEEE Transactions on Ultrasonics, Ferroelectrics, and Frequency Control, 43, pp. 509-518.

KANANI, B. and BURDESS, J. (1990). The piezoelectric cylinder gyroscope, in Proceedings of the Institution of Mechanical Engineers, Cambridge, UK, pp. 61-66.

LANGDON, R. (1982). The vibrating cylinder gyro. The Marconi Review, 46, pp. 231-249.

LANGMAID, C. (1996). Vibrating structure gyroscopes. Sensor Review, 16, pp. 14-17.

LeisSA, A. (1973). Vibration of Shells, National Aeronautics and Space Administration, Washington.

Loveday, P. (1996). A Coupled Electromechanical Model of an Imperfect Piezoelectric Vibrating Cylinder. Journal of Intelligent Material, Systems, and Structures, 7, pp. 44-53.

NAYFEH, A. H. and MOOK, D. T. (1979). Nonlinear Oscillations, Wiley-Interscience, New York.

NAYFEH, A. H. and RAOUF, R. (1987). Non-linear Oscillations of Circular Cylindrical Shells. International Journal of Solids and Structures, 23, pp. 1625-1638.

NAYFEH, A. H. and RAOUF, R. (1987). Nonlinear Forced Response of Infinitely Long Circular Cylindrical Shells. Journal of Applied Mechanics, 54, pp. 571-577.

NAYFEH, A. H., RAOUF, R. and NAYFEH, J. (1991). Nonlinear Response of Infinitely Long Circular Cylindrical Shells to Subharmonic Radial Loads. Journal of Applied Mechanics, 58, pp. 1033-1041.

Shatalov, M., DU PRE Le RouX, J. and KoCH, F. (1996). Estimation of vibratory gyroscope parameters with data derived for the vibrating element, in DGON Symposium on Gyro Technology, Stuttgart, Germany.

ShutA, K. and ABE, H. (1995). Compact Vibratory Gyroscope. Japanese Journal of Applied Physics, 34, pp. 2601-2603.

SOEDEL, W. (1993). Vibrations of Shells and Plates, Marcel Dekker, New York.

YASUDA, K. and KUSHIDA, G. (1984). Nonlinear Forced Oscillations of a Shallow Spherical Shell. Bulletin of the Japanese Society of Mechanical Engineering, 27, pp. 2233-2240. 